\title{
Pneumatically adaptive light modulation system (PALMS) for buildings
}

\section{Citation}

Hinz, K., J. Alvarenga, P. Kim, D. Park, J. Aizenberg, and M. Bechthold. 2018. “Pneumatically Adaptive Light Modulation System (PALMS) for Buildings." Materials \& Design 152 (August): 156-167. doi:10.1016/j.matdes.2018.04.044.

\section{Published Version}

doi:10.1016/j.matdes.2018.04.044

\section{Permanent link}

http://nrs.harvard.edu/urn-3:HUL.InstRepos:37235085

\section{Terms of Use}

This article was downloaded from Harvard University's DASH repository, and is made available under the terms and conditions applicable to Open Access Policy Articles, as set forth at http:// nrs.harvard.edu/urn-3:HUL.InstRepos:dash.current.terms-of-use\#OAP

\section{Share Your Story}

The Harvard community has made this article openly available.

Please share how this access benefits you. Submit a story.

Accessibility 
and Design

Manuscript Number: JMAD-D-17-07859R2

Title: Pneumatically Adaptive Light Modulation System (PALMS) for Buildings

Article Type: Research Paper

Keywords: Optically adaptive materials,

Micro-scale surface structures,

Light transmittance design,

Responsive glazing systems,

Pneumatic actuation systems,

Pneumatic building envelope design.

Corresponding Author: Mr. Kevin Hinz, MArch

Corresponding Author's Institution: Havard University Graduate School of Design

First Author: Kevin Hinz

Order of Authors: Kevin Hinz; Jack Alvarenga, BS, MS Physics, Material Science, Engineering; Philseok Kim, BS, MS, PhD, Physical Chemistry; Daekwon Park, MArch, MDes; PhD Design Technology; Joanna Aizenberg, BS, MS Chemistry, Ph.D. Structural Biology; Martin Bechthold, DiplomIngenieur, PhD Digital Fabrication

Abstract: This research introduces a novel approach to control light transmittance based on flexible polydimethylsiloxane (PDMS) films that have been plasma-treated such that micro-scale surface features have a visual effect as the film responds to applied strain. The effect is continuously tunable from optically clear (71.5\% Transmittance over a 400-900 nm wavelength) to completely diffuse (18.1\% T). Changes in the film's optical properties are triggered by bi-axial strains applied using a pneumatic system to form pressurized envelopes. This paper reports on a series of experimental studies and provides system integration research using prototypes, simulations and geometric models to correlate measured optical properties, strain, and global surface curvatures. In conclusion, a design is proposed to integrate PDMS light control within existing building envelopes.

Two alternatives are investigated and compared: System A uses positive pressure featuring an exterior grid to restrain and shape the inflated film during expansion; System B uses negative pressure where the films are shaped according to the geometry of an interstitial grid that serves as a spacer between two film surfaces. Both systems can provide effective control of opacity levels using pneumatic pressure and may be suitable for incorporation with existing glazing systems or ethylene tetrafluoroethylene (ETFE) pneumatic envelopes. 


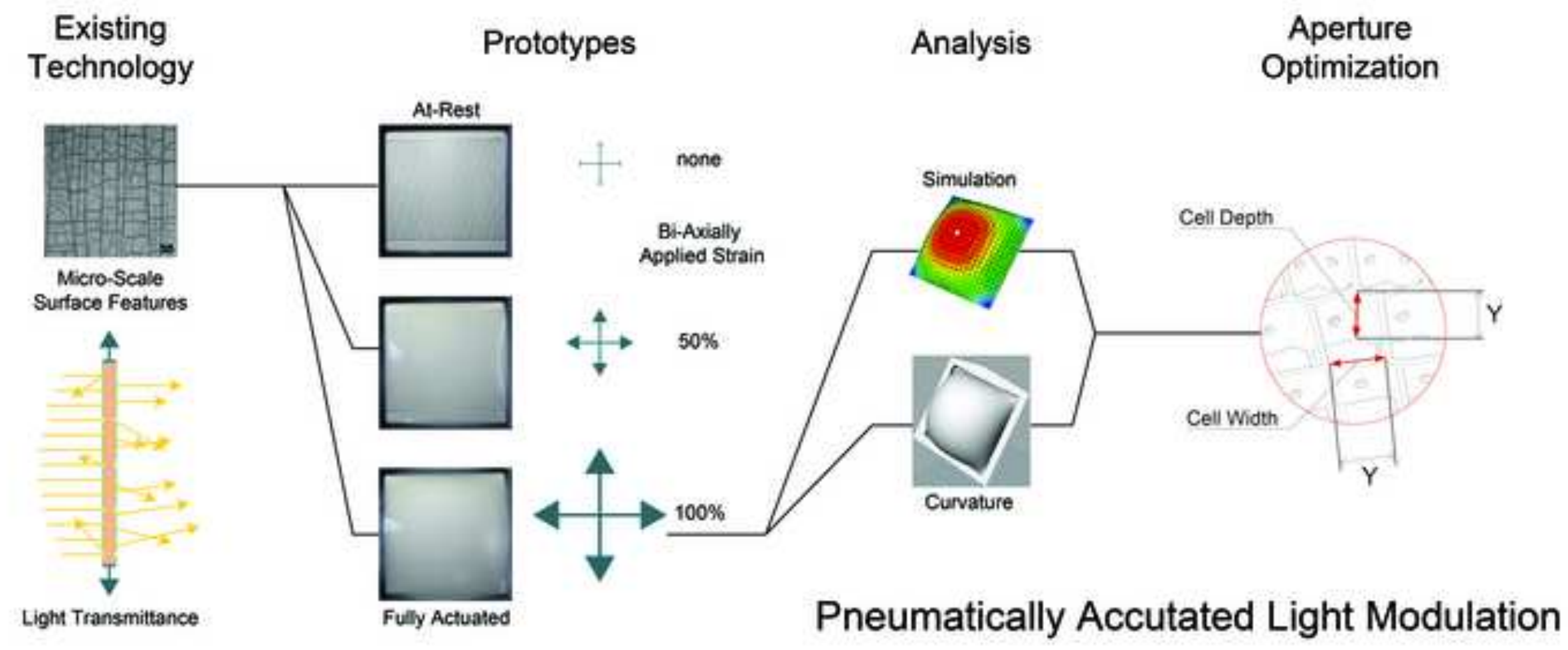




\title{
Pneumatically Adaptive Light Modulation System (PALMS) for Buildings
}

\author{
Hinz, K.a,*, Alvarenga, J.b, Kim, P.b, Park, D. a, Aizenberg, J.b, Bechthold, M. a \\ aHarvard Graduate School of Design, 48 Quincy St., Cambridge, MA 02138, USA \\ bWyss Institute for Biologically Inspired Engineering at Harvard University, 3 Blackfan \\ Cir., Boston, MA 02115, USA
}

\section{Abstract}

This research introduces a novel approach to control light transmittance based on flexible polydimethylsiloxane (PDMS) films that have been plasma-treated such that micro-scale surface features have a visual effect as the film responds to applied strain. The effect is continuously tunable from optically clear (71.5\% Transmittance over a 400-900 nm wavelength) to completely diffuse $(18.1 \% \mathrm{~T})$. Changes in the film's optical properties are triggered by bi-axial strains applied using a pneumatic system to form pressurized envelopes. This paper reports on a series of experimental studies and provides system integration research using prototypes, simulations and geometric models to correlate measured optical properties, strain, and global surface curvatures. In conclusion, a design is proposed to integrate PDMS light control within existing building envelopes.

Two alternatives are investigated and compared: System A uses positive pressure featuring an exterior grid to restrain and shape the inflated film during expansion; System B uses negative pressure where the films are shaped according to the geometry of an interstitial grid that serves as a spacer between two film surfaces. Both systems can provide effective control of opacity levels using pneumatic pressure and may be suitable for use with existing glazing systems or ethylene tetrafluoroethylene (ETFE) pneumatic envelopes.

Keywords: Optically adaptive materials, Micro-scale surface structures, Light transmittance design, Responsive glazing systems, Pneumatic actuation systems, Pneumatic building envelope design.

Highlights:

- The development of an adaptive film is designed to dynamically control light and views in buildings;

- Pneumatic pressure is found to successfully activate the adaptive film;

- Aperture geometry and pneumatic pressures both affect the distribution of strain and level of visibility control through the adaptive film;

- Predictable relationships between the film's surface curvature and optical performance exist;

- Continuously tunable light control can be achieved when the adaptive film is combined with existing envelope technologies; 
Funding:

Harvard University Graduate School of Design, Wyss Institute for Biologically Inspired Engineering at Harvard University

\section{INTRODUCTION}

\section{Dynamic Privacy and Light Control in Buildings}

The control of privacy in glazing envelope systems has been a goal since the early uses of glass in buildings, but at the present, there are no technically satisfying solutions that are fully adaptable and simultaneously cost effective. An alternative to glazed envelopes are pneumatically pressurized ethylene tetrafluoroethylene (ETFE) film' pillow' facades; these systems have few options to control light and privacy. Static glazing systems such as textured glass, cast glass panes, colored glass and more recently, fritted or etched glass panes, allow for privacy while maintaining light transmittance. However, static systems offer steady-state light control and do not adapt to changing requirements that might originate from programmatic and functional needs. Mechanically activated dynamic light control systems exist but are slow to react to environmental conditions [1] [2]. Solutions such as blinds, shades, and curtains can reduce light levels and provide privacy, but mechanically actuated dynamic shading and privacy systems are not very robust, require regular maintenance, and are difficult to deploy at a large scale. The research presents new experimental insight on the optical properties of doubly-curved PDMS film, correlating light transmittance with simulated bi-axial strains using non-linear finite element analysis. The paper also presents various prototypes and proposes an initial design solution for integrating a Pneumatically Adaptive Light Modulation System (PALMS) into existing roof systems and building facades, with a focus on pneumatic ETFE systems.

\section{1. The Need for Privacy and Light Control: Existing Adaptive Technologies}

The most common dynamic envelope control systems for buildings include mechanically actuated layers of internal or external shading elements. These systems feature a myriad of moving parts and are complex to design, operate and maintain [1] [2]. Pneumatically actuated composite and soft microfluidic materials designed for light transmittance control have been presented by Jimnez (2016) and Uar (2012) but few combinations permit a continuously adjustable transition from completely clear to opaque [3] [4]. Several electronically activated solutions exist, where the switching of glazing systems between clear and translucent is easily achieved [5].

Electrochromic (EC) glazing systems are commercially available and find use in buildings, usually applied as a thin sandwich layer on top of a single layer or between two layers of a glass substrate. The EC layer consists of two transparent electrical conductors separated by a ceramic metal oxide coating and are switched on and off through the application or absence of low voltage [6]. Simulations analyzing the thermal effects of EC films suggest that significant energy reductions are possible only when films are applied to the outside of a glazed envelope, i.e. on a building's exterior (in contrast to the interior side of the glazed envelope) [7]. Additionally, it is important that the substrate and EC layer have a similar thermal expansion coefficient, thereby reducing stress on the EC film over the life of the window [8].

Other systems such as polymer-dispersed liquid crystal (PDLC), encapsulated liquid crystal (NCAPnematic curvilinear aligned phase), and guest-host liquid crystal, use dispersed liquid crystal technologies to affect change in translucency [9]. Liquid crystal molecules are diffused into a polymer matrix, forming droplets as the matrix solidifies. When subject to an electric field 
between two electrode layers, the once randomly aligned liquid crystals change orientation to align with the electric field, switching from cloudy to clear in the absence-of or application-of voltage. Electrophoretic or Suspended Particle Devices (SPD) systems are similar to liquid crystal technologies, featuring an electronically switchable thin layered film $(0.025 \mathrm{~mm})$ that is applied to existing planar substrates [10]. SPD light transmittance ranges between $5 \%$ and $90 \%$ are possible. Costs are high for these devices, starting at about $\$ 800 / \mathrm{m} 2$, an expense reflecting capital-intense production methods and relatively low sales volumes with little market penetration [11]. Consumer applications are limited because few companies are dedicated to the design, application and utilization of finished SPD products. ' Smart' glazing systems are expensive to manufacture and install, and have design parameters most often limited to flat glazed substrates [9]. Numerous dynamic light control technologies for standard glazed envelopes are easily accessible but few systems are available at a reasonable cost [5].

Paraffin based Phase Change Materials (PCM) used in glazing systems may also provide a level of adaptability, changing from translucent (in the solid state) to completely transparent (when melted) [12]. The typical PCM window unit is assembled from two panes of clear glass surrounding a layer of paraffin wax. As the wax moves from liquid to solid, it affects the optical and thermal performance through a building's envelope. From the given literature, PCM appears to have a significant affect on the exchange of energy and on thermal comfort, but with little improvement in visual comfort for an interior space [13] [14].

The material palette for transparent envelopes is expanding to include pneumatic facades using ETFE films. The most common are positive pressure systems whereby two layers of thin ETFE films expand toward the outside of the component resulting in 'pillow' - like shapes, stabilized by a pressure differential between the interior cavity and the atmosphere. One existing solution for light transmittance control in buildings features two or more interior layers of patterned film that can be pneumatically moved close together or far apart [15]. Each layer is designed such that when the cavities are inflated and the layers are spaced apart, the patterns misalign to permit or deny views and regulate the penetration of sunlight. When reversing the interior pressure, the two patterned ETFE layers are brought into immediate adjacency such that alignment of the respective figures are inverted to control glare and solar gains. Typically, only two extreme pressure extremes are possible, and the patterns always remain visible, obstructing views even in the most open (e. g. inflated) configuration [16].

\section{(a) Prototype A2: at rest (b) Prototype A2: actuated Figure 1: System A, Positive Pressure}

\subsection{PALMS as an alternative solution}

PALMS is a Pneumatically Adaptive Light Modulation System that deploys an inexpensive adaptive film in combination with a pneumatic actuation system to allow for privacy and solar control of building envelopes. The system uses pneumatic pressure to apply bi-axial strain to an optically clear, 0.254 $\mathrm{mm}$ thick PDMS film (F1). The stretched films reveal micro-scale surface cracks, an effect of controlled oxygen plasma exposure (F2). As the micro-scale cracks in the oxidized layer expand, light is scattered and opacity is reduced. The system allows for a controlled gradation of light transmittance while simultaneously achieving visual privacy control from clear to fully obstructed views.

Bi-axial strain is generated in the PDMS film as it expands in response to compressed gas (System A) or to negative atmospheric pressure (System B). The strained films reduce the amount of light being transmitted and also obstruct views for privacy. The term actuation' is used to describe the oxidized PDMS film when strained (in this case pneumatically), and 'full actuation' is 
defined as the state of strained oxidized film having the lowest possible light transmittance level within a specific aperture cell.

\section{3. Adaptive Actuation of Plasma-Treated PDMS Films: Fundamental Behavior}

Bi-axially strained plasma-treated PDMS films generate a reversible, micro-scale surface structure that can be used for adaptive privacy and light control systems (F2). Yang, et. al. found that the micro-scale surface geometry resembles a one-dimensional distraction grating as a result of compressive stress (e.g. from a mechanical strain) created through a Poisson-ratio effect across a stretched thin film [17]. Testing of oxidized PDMS subject to linear strain was also reported by Kim et.al. (2013), their experiments showed that continuous control of visible light transmittance from $0 \%$ to nearly $90 \%$ at normal incidence was achieved with strains ranging from 0-50\% [18]. It is known that a resulting periodic surface structure is formed in plasma treated PDMS, scattering and diffusing light with applied strain, thereby substantially reducing light transmittance in a controlled manner.

To maximize the affect of scattered light for use in buildings, plasma dose and treatment times of test samples were exaggerated for use in our experiments. The increased treatment times revealed hierarchical surface crack patterns, fissures in the oxidized PDMS surface that when similarly stretched, more greatly affect light transmittance to a level easily perceived by the human eye. Integrating PDMS into the design of buildings, or its use in other industries, has not been extensively studied beyond novel light control systems based on microfluidic networks and flexible pneumatic louvers [4] [19]. Intrinsic elasticity, modulus, and the optical qualities of oxidized PDMS film makes it an ideal complement to ETFE or similar facades, and a suitable standalone privacy system for building interiors.

PDMS silicones are a common encapsulate material for photovoltaics, owing to their excellent resistance to oxygen, ozone, UV light, and insensitivity to moisture [20, 21]. In addition, PDMS is a very fire-resistant material and prevalent in the active research area of soft sensors and soft electronics for its long term mechanical stability among other material properties [22, 23] [24]. For an elastomeric polymer strained within the domain of elastic deformation (up to 100\% elongation with PDMS rubber), mechanical fatigue is not expected nor was observed over the course of our experiments.

\begin{tabular}{|c|c|c|}
\hline Material & ETFE & PDMS \\
\hline Tensile Strength [MPa] & $38-48$ & 5.5 \\
\hline Elongation [\%] & $100-350$ & 250 \\
\hline Thermal Conductivity $[\mathrm{W} / \mathrm{m} \cdot \mathrm{K}]$ & 0.238 & 0.21 \\
\hline Total Transmittance & $93.8+/-0.1$ & $97.00 \%$ \\
\hline Haze & $9.2+/-0.8$ & \\
\hline Cost (film sheets) & $\begin{array}{l}0.127 \mathrm{~mm} \text { thickness: } \$ 327 \mathrm{USD} / \mathrm{m}^{2} \\
{[25]}\end{array}$ & $\begin{array}{l}0.508 \mathrm{~mm} \text { thickness: \$258USD / } \\
\qquad \mathrm{m}^{2}[26]\end{array}$ \\
\hline
\end{tabular}

Table 1: Material property comparison between ETFE and PDMS films [27].

Figure 2: Micro-scale surface cracks on plasma-treated PDMS film. Left: Photographs of micro- scale surface features of pneumatically unstrained and strained plasma-treated film. Top Left: sample at rest; Bottom Left: actuated sample. Right: Illustration of pneumatically strained plasma treated PDMS diagramming light scattering optical effect. 


\section{Prototype Design Studies}

A primary challenge in the use of oxidized PDMS film for light transmittance control is the need for relatively large strains of $20-30 \%$ required to affect change in optical properties [18]. When used as a flat film integrated into facade systems, strains can be induced by rolling the film onto cylindrical features similar to those used in roller-blinds. This solution leads to bulky mechanical elements along the top or the side of the window frame, and the need for lateral guide rails to restrain the film' s edge. The forces required to actuate treated PDMS can be significantly larger than what is present in commercially available roller blinds, requiring mechanically robust and thus costly hardware. In addition, large scale wrinkles in the PDMS film are particularly difficult to avoid with roller solutions; wrinkles often occur in mechanically strained PDMS film because of its large Poisson ratio (up to 0.5), forming along the direction of pull, parallel to the applied stress [28]. These material characteristics make this application difficult for small glazed surfaces at best, and prohibitive for large glazing or pneumatic ETFE surfaces found in contemporary buildings where privacy and light control are increasingly unresolved.

\section{(a) System A: Positive Pressure (b) System B: Negative Pressure} Figure 3: System Prototypes

\section{1. System Design Principles}

Two alternative actuation methods (F3) were employed in our research to achieve the strains needed for a significant change in optical transmittance, providing new insight into the relationship between bi-axial strain and curvature for actuating thin plasma-treated PDMS films. Both actuation methods are pneumatic: one operating with positive pressure (System A) and the other with negative pressure (System B). The resulting applied strain of the PDMS film is bi-axial and free from large scale wrinkles in either application.

Pressures can be dynamically tuned in either system A or B using gate valves that control the exchange of pneumatic fluid. Experiments with System A showed that a pneumatic pressure less than $10.3 \mathrm{kPa}$ is required to fully actuate well balanced apertures when using the $0.254 \mathrm{~mm}$ film.

Depending on the success of the vacuum seal between System B experiments, negative pressures up to $177.8 \mathrm{~mm} \mathrm{Hg}$ were required to achieve full activation using the same $0.254 \mathrm{~mm}$ film.

\section{1. 1. System A: Positive Pressure}

\section{Figure 4: System A section. Above: System A, at rest. Below: System A, actuated}

System A includes two layers of a tightly secured PDMS film held in place along its edges by a perimeter frame pressing against a PTFE gasket (F4). The interstitial space contains a compressible fluid (in this example, gas) that when increased with respect to atmospheric pressure, forms a ' pillow' -like inflated shape; bi-axial strains are pneumatically introduced into the film. As the film expands in two dimensions, micro-scale cracks appear on the surface of the treated PDMS (F2), affecting light transmittance through the once transparent film. To limit the height of the ' pillow', additional physical constraint grids are used on the exterior of the films. These grids can be readily shaped from flat material stock, forming ornamental, cell-1ike patterns. Significant design differences between prototypes (A1-4, Fig 8) include aperture proportions in the applied grid, experimental plasma treatment schedules (for the PDMS films) and improved seal design to more efficiently actuate the test samples.

\subsubsection{System B: Negative Pressure}




\section{Figure 5: System B section. Above: System B, at rest. Below: System B, actuated}

System B includes two layers of PDMS film that form around an interstitial spacer grid when a negative pressure is applied to the expansion cavity; films and grid cells are contained within the depth of a perimeter frame (F5). Perforations between cells of the interstitial layer provide a continuously interconnected pressure chamber. Due to the vacuum pressure, the connection between film and frame is self-sealing. In our experiments, an unregulated pump pulls fluid from the interstitial layer, expanding the PDMS film into the cell chamber in a similar ' pillow' -like form as observed in System A. The interstitial grid, subject to compression, equilibrates the tensile stress in the film and controls the size of the ' pillow' to maximize the application of bi-axial stress and subsequent creation of surface micro-cracks. The grid can take on a range of shapes that add to the overall cell-like appearance of the system. If opposing films collide, local strains are reduced thereby increasing light transmittance in the areas of contact. The most significant design difference between prototypes (B1-B2) include the variable chamber depth in B2, a design feature introduced to minimize collisions between opposing films (Fig 9a-b).

\section{2. Prototype Construction and Material Aspects}

Each prototype was $300 \mathrm{~mm}$ x $300 \mathrm{~mm}$ in size and composed of similar components: a perimeter frame to create the pneumatic chamber and accept the other components, two PDMS films that enclose a sealed airspace, hardware and retention bars to mechanically restrain the film during assembly, a constraining layer (either interstitial or constraining grid), and various pneumatic fittings (F6). Figure F7 shows 9 alternative patterns for the constraining grid.

\section{Figure 6: System A typical assembly}

To establish initial design guidelines, multiple prototypes for System A and System B were produced and optically tested. The assemblies used industrially manufactured PDMS films $0.254 \mathrm{~mm}$ and 0.762 $\mathrm{mm}$ thick that were exposed to oxygen plasma over periods ranging from 400 to 2400 seconds. Film samples measuring approximately 320 x $320 \mathrm{~mm}$, were placed in a Plasma-Etch PE-200, with a $13.56 \mathrm{MHz}$ $\mathrm{RF}$ generator operating at $250 \mathrm{~W}$ with $20 \mathrm{sccm} 02$ flow and base pressure of 180 mTorr.

The geometric composition of shapes in the constraining grid of all prototypes explore a number of opening and aperture edge conditions, illustrating the range of opacity gradients visible in regular and elongated apertures (F7-F9). Early examples used a small hand pump to actuate the prototypes, later tests incorporated a pneumatic source controlled via a micro pressure fluid regulator. Standard push-to-connect pneumatic tubes and fittings complete a circuit between the pneumatic source and expansion chamber.

Figure 7: Constraining grid array

\section{2. 1. System A Prototypes (positive pressure)}

Prototype A1 uses positive pressure to investigate basic optical properties when using variable, irregularly shaped apertures in the constraining grid (see also F3a). The component system includes a 3D-printed constraining layer, a single layer of plasma-treated film, a perimeter frame and expansion chamber with an acrylic backing plate to seal the chamber. Developments integrate a PTFE gasket to compress the film between the constraining layer and the expansion chamber, significantly improving fluid retention.

Figure 8: System A Prototypes. Clockwise from top left: A1, A2, A3, A4 
Prototype A2 has an improved section design that includes a uniform perimeter frame to easily accept interchangeable constraining grids, illustrating the range of possible architectural affects (F7). The constraining grid test layers included $3.175 \mathrm{~mm}$ cold rolled steel and $1.28 \mathrm{~mm}$ stainless steel with $44 \mathrm{~mm}$ and $95 \mathrm{~mm}$ parallelogram-shaped apertures using $3 \mathrm{~mm}$ and $6 \mathrm{~mm}$ gridded mullions. Both material types successfully sealed and constrained the PDMS film. Gasketed assemblies were fully actuated with less than $10.3 \mathrm{kPa}$ and maintained pressure for more than 60 minutes before $\mathrm{re}^{-}$ pressurization was required. Noticeable deflections were present in the constraining grid test assemblies; $3.175 \mathrm{~mm}$ cold rolled steel with a $95 \mathrm{~mm}$ aperture and $3 \mathrm{~mm}$ mullion served to best maximize treated film actuation while minimizing grid distortion (see also Fla-b).

Prototype A3 evaluates alternative film retention strategies and tests a novel fabrication process with a permanent silicone-based pneumatic seal. The perimeter frame and sealing gasket is manufactured as a single, multi-material 3D print. The PDMS film is held in place by metal retention bars while a liquid silicon gasket is poured over the edge of the film where it meets the pneumatic chamber. A series of fibrous strings are wrapped around the assembly to restrict the expanding film, facilitating full actuation of the treated film and limiting expansion depth.

Advantages include an integrated manufacturing process, minimal structural dimensions for a typical assembly (maximizing light transmission) and a fibrous constraining grid that reduces the visual impact of a glazed assembly.

Prototype A4 expands the assembly of Prototype A2 into an array of $2 \times 2$ units, equating to four expansion chambers and four pairs of PDMS films retained by a single constraining grid. Prototype A4 is problematic to assemble and the chamber seals were less than satisfactory although leaks in the pneumatic chamber were overcome with an increased flow of pneumatic fluid.

\section{a) Prototype B2 at rest (above) and actuated (below) (b) The cell-width and cell-depth ratio is ideally 1:1}

\section{Figure 9: Prototype B2, Negative Pressure}

\subsubsection{System B Prototypes (negative pressure)}

Prototype B1 \& B2 integrate the pneumatic chamber and constraining grid into an interstitial layer; perforations between adjacent cells establish a continuous vacuum cavity. The principal design advantage of System B includes a significant reduction of the sectional depth in fully actuated assemblies thereby reducing PALMS' $s$ impact on the depth of the window cavity to better facilitate the system's integration into a pneumatic pillow envelope. In addition, negative pneumatic pressure contributes to the self-sealing capabilities of PDMS and requires only a simple retention frame to hold the film in place, reducing mechanical complexity and assembly time.

In Prototype B1, the interstitial layer is a 3D printed hexagonal grid with $8.3 \mathrm{~mm}$ radius aperture cells. Prototype B2 uses a 3D voronoi grid cell pattern, a modification of the hexagonal grid, to offer a compelling solution while illustrating design opportunities to control light transmittance with a range of aperture cell sizes in a single prototype (F9). B2 cell depths directly correspond to cell widths across the minor span, an offset sectional dimension of the interstitial frame in accordance with an aperture radii to expansion depth ratio established in earlier experiments (see ch. 4). In these examples, the depth of the interstitial frame is approximately the width of the aperture. The variable depth creates a local pneumatic cell chamber deep enough for the film to fully actuate before coming into complete contact with its opposing film. 


\section{Bi-axial strained PDMS: Optical Properties and Computational Analysis}

Yang et.al. demonstrated that strain-induced buckling on plasma- treated PDMS can be expanded from 1D to 2D patterns by introducing bi- axial strain mechanisms that allow a variety of different surface patterns including 2D herringbone surface structures [17] [29]. We visually observed a transmittance gradient across the surface of the PDMS' pillow,' producing affects similar to those described in previous research [17] [18]. Plasma treatment times directly correlate with the oxidized layer thickness (Kim, et.al, 2013), which in turn determines crack amplitudes, pitch and ultimate fissure depth; these surface features modify visual light transmittance levels. Idealized plasma treatment schedules were established as described in $\mathrm{CH} 2.2$ to minimize the presence of surface distortions in an at rest film while maximizing opacity in fully actuated samples.

\section{Figure 10: Spectrometer set-up diagram}

Figure 11: Prototype A5, $10 \mathrm{~cm} 2$ aperture

A $10 \mathrm{~cm}^{2}$ aperture prototype (Prototype A5) using two $0.254 \mathrm{~mm}$ PDMS sheets treated for 1200 seconds was fabricated to measure light transmittance levels and to compare physical observations results (F10-11). It is important to note that only the physical visible light spectrum was analyzed to narrow the scope of focus at this early stage of research. when investigating design applicationsfor architecture.

Transmissions were recorded at four positions (see F12: 0,$0 ; 0,3 ;-3,3 ;-3,0$ \& T1) through a relaxed and fully actuated (at $10.3 \mathrm{kPa)}$ ) test aperture. Light transmittance passing through Prototype A5 was recorded with an 0cean 0ptics USB2000+VIS-NIR spectrometer (350-1000 nm wavelength range with $1.5 \mathrm{~nm}$ optical resolution) in conjunction with an tungsten halogen light source (Model LS-1-LL, Ocean Optics, spectral range 360-2500 nm) coupled to a 400 um diameter fiber optic cable. A collimating lens (Model 74-VIS 0cean Optics) and variable pinhole aperture limited incoming stray light and maintained a constant normal incidence spot size of $\sim_{2} \mathrm{~mm}$. The transmitted light was collected and focused onto a second fiber optic cable using a $50 \mathrm{~mm}$ diameter collection lens with a focal length of $60 \mathrm{~mm}$ behind the PDMS sample. Individual spectra were collected with an integration time of $2000 \mu \mathrm{s}, \mathrm{n}=3$ averaging and boxcar smoothing.

Percent transmittance (\%) is calculated as the ratio of the intensity of light leaving the sample (I, in 'Counts' as measured by the spectrophotometer) over the intensity of light entering the sample $\left(I_{o}\right)$ multiplied by 100. In our case the light entering the system is measured with the sample removed from the holder under the same ambient (low light) conditions and acquisition parameters. Acquisition parameters are pre-determined to ensure detector signal is not saturated. A "dark spectrum" is collected in ambient conditions with light source turned off prior to measurements for baseline normalization. The background spectrum was collected without turning on the light source and all the spectra collected were normalized by a spectrum collected without placing a sample in the beam path (i.e. 100\%T). A ratio of the detected light intensity (at normal incidence) is then taken with the sample in place and at different stages of mechanical deformation to calculate a percent transmittance. A mean value of this transmittance across the 400-900 nm wavelength range is reported for simplicity in comparing the light magnitude since the effect is largely wavelength-independent in this range. The results show that light transmittance is distributed as a contoured function and that strain distribution closely correlates with light transmittance (F12a-b).

Prototype $\mathrm{A5}$ (inflated to $10.3 \mathrm{kPa}$ pressure) was then digitally scanned immediately following the light transmittance recording to compare the relationship between curvature and opacity. Immediately following the light transmittance recording, Prototype A5 (still inflated to 10.3 kPa) 
was digitally scanned using a David SLS-3, 3D Structured Light 3D scanner [30]. The David software version 3.10.4 was used to generate a composite mesh with 339, 139 polygons, digitally representing the $10 \times 10 \mathrm{~cm}$ inflated pillow and $8 \mathrm{~mm}$ of the immediately adjacent constraining grid. The final mesh resolution is calculated to $+-0.06 \mathrm{~mm}$ at $0.05 \%$ of the total scan size per manufacturer specification. Observations Transmittance measurements show that full actuation is realized when a similar 'pillow' height dimension (or surface curvature radii) and aperture radii are present (approaching 1:1); in this case, an expansion height of $440 \mathrm{~mm}$ was recorded on the $10 \mathrm{~cm}^{2}$ test prototype.

(a) Photograph of light transmission of an actual sample (b) Strain simulation, showing max in-plane strain.

Figure 12: Fully Actuated System: Actual to Simulation Comparison

\begin{tabular}{|c|c|c|c|c|}
\hline Sample State & Position & $\begin{array}{c}\text { \% Transmittance } \\
\mathbf{( 4 0 0 - 9 0 0 n m ~ m e a n ~ v a l u e ) ~}\end{array}$ & $\begin{array}{c}\text { Min In-Plane } \\
\text { Logarithmic Strain }\end{array}$ & $\begin{array}{c}\text { Max In-Plane } \\
\text { Logarithmic Strain }\end{array}$ \\
\hline Actuated & $(-3,3)$ & 18.8 & $2.40 \mathrm{E}-01$ & $3.20 \mathrm{E}-01$ \\
\hline Actuated & $(-3,0)$ & 19.1 & $3.20 \mathrm{E}-01$ & $3.90 \mathrm{E}-01$ \\
\hline Actuated & $(0,3)$ & 21.6 & $3.20 \mathrm{E}-01$ & $3.90 \mathrm{E}-01$ \\
\hline Actuated & $(0,0)$ & 18.1 & & $4.10 \mathrm{E}-01$ \\
\hline Relaxed & $(-3,3)$ & 71.7 & & \\
\hline Relaxed & $(-3,0)$ & 71.5 & & \\
\hline Relaxed & $(0,3)$ & 69.1 & & \\
\hline Relaxed & $(0,0)$ & 67.5 & & \\
\hline & & & & \\
\hline & & & & \\
\hline
\end{tabular}

Table 2: Transmittance measurements of A5 when fully actuated (see F12a-b). Note the change in a viewers ability to fully recognize shapes (in the case of Fig 12a, vertical lines near position -3,3) through a PDMS sample at rest and while fully actuated.

To understand the strain distribution in the proposed pneumatic system, non-linear finite element analysis was conducted using Abaqus, a commercially available analysis software with a proven ability for geometrically non-linear analysis. Simulations of the square aperture suggest that pneumatically actuated PDMS experiences an anisotropic effect of bi-axial strain, and that strain is distributed as a contoured function of applied force (F12b). These results were verified by the spectrometer measurements using prototype A5. Visual observations (of the test prototype) confirm the presence of an opacity gradient across the film surface, a gradient corresponding to the simulated strain contours across an aperture cell (F12a). Transmittance is increased at the corners, a result of a localized strain reduction as evident in the simulation.

RESULTS

\section{Relationships between optical properties and aperture geometry}

This report focuses on the application of an existing technology and its predictable application for user experience in building environments. Measurements and simulations confirm a direct relationship affecting light transmittance existing between: plasma treatment time, applied strain, aperture geometry and surface curvature. The most opaque areas of a fully actuated film correlate to areas of the highest strain and possess nearly the same 1:1 ratio of curvature-to-aperture radii. 


\section{1. Plasma Treatment}

PDMS Samples that were plasma-treated for $<400$ seconds show little change in translucency. Samples treated for $>2400$ seconds developed iridescent reflections and other visible distortions, a photoelastic phenomenon likely resulting from heat generated within the plasma chamber during exposure [31]. The heat effectively expands the PDMS film and keeps it in a pre-stretched state thereby increasing the area of surface exposed to oxidization. Once the sample is removed from the plasma chamber, the PDMS film cools and shrinks, placing compressive stress on the oxidized surface resulting in spontaneous surface buckling or cracking, without apparent externally applied strain. As a result, iridescent reflections are revealed (while in a relaxed state). The PDMS sheets used in the Prototype A5 spectrometer test were treated for 1200 seconds, a treatment time experimentally determined to be the ideal balance between surface clarity (when at rest) and maximum opacity (when fully actuated).

\subsection{Applied Strain}

Aperture geometry affects the distribution of applied strain for a given pressure differential. Because a 1:1 aperture radii-to-expansion depth is required to produce the necessary bi-axial strains, maximum actuation is effectively limited by the smaller of the two aperture radii (F13left column, as shown by dimension X). This level of maximum expansion (for a given pressure) is proportional to the minor radii of a given aperture. Long narrow grid proportions require more pressure to achieve the required strains for full actuation (F14-top), compared to pressure required to fully actuate nearly square grid shapes (F14-bottom).

Figure 13: Aperture width to radii of curvature: Left column: elongated aperture. Right column: equal proportioned aperture Aperture proportions largely determine the degree to which uniform strains develop in the inflated condition. Ideal conditions are present in a circular opening, whereas elongated apertures generally expand to a max height dictated by the minor dimension. This reduces force in one dimension of the pneumatically applied bi-axial strain for a given pressure thereby reducing effective change in opacity.

\section{3. Aperture Geometry and Design}

Both systems A \& B rely on a constraining grid to locally control the expanding film and applied strain; this grid creates ornamental patterns that adds design interest to the system. The grid determines the overall appearance of the system and also affects strains across one or more apertures thereby directly effecting the optical properties of actuated film within each aperture (note varied levels of actuation shown in F3a). It is important to note that aperture shape does not have as profound an affect on a sample's ability to achieve full actuation as does aperture proportion; the number of sides a shape is inconsequential (to achieve full actuation) but the strain required to achieve full actuation is determined in part by the aperture's width:length ratio.

Figure 14: Early shape experiments at $117.2 \mathrm{kPa}$ Relationship between aperture proportion and opacity. Above: Long-narrow proportion. Below: Near equal length-width proportion.

A direct relationship between grid proportion and light transmittance levels exists. Shapes with a near equal length to width ratio provide the greatest range of translucency control for a given pressure differential (see F13). Because aperture geometry directly affects the resulting strains, irregularly designed constraining grid arrays produce predictably gradated light transmittance and visual privacy control. The results can be observed in Prototype Al as a gradated opacity within a single aperture (F3a \& F8-top left). 


\section{4. Surface Curvature}

Surface curvature analysis of the 3D scanned digital model representing Prototype A5 when fully atuated (inflated to $10.3 \mathrm{kPa}$ ), reveals near spherical geometry at the apex; the principle curvature of the pillow increases near the aperture edge. Opacity is at its greatest when principle curvature radii are near equal to aperture radii (along the same plane of principle curvature), a point typically near the apex. Conversely, opacity decreases near the edge of the ' pillow, where a decrease in curvature exists at the intersection between the film and the constraining grid.

When shapes have long-narrow proportions or apertures are irregularly shaped, a film' s expansion depth for a given pressure differential is limited by the minor aperture radii; conversely, the proportion between the major radii and the expansion depth will dictate the greatest possible opacity (for the given pressure differential). These results substantiate visual observations of an ideal 1:1 aperture radii (along the greatest distance across) to expansion depth ratio of the constraining grid in early experiments. The grid cell depth of Prototype B2 is designed with this observation in mind, providing a framework to restrict the opposing films from touching and canceling the pneumatically applied strains (see F9).

\subsection{Design Variables affecting light transmittance in both systems}

(1) The duration of PDMS oxygen plasma treatment affects the depth of surface oxidization: The treatment times affect the thickness of the oxidized layer and directly correlate to the optical properties of the modified material. Experiments show that plasma intensity and exposure intervals can be optimized for the highest dynamic contrast derived from micro-scale cracking during sample actuation.

(2) The applied strain is a function of the pressure differential: The pneumatic pressure for Systems A \& B need to create appropriate strains in the PDMS film. Dynamic pressure controls can be integrated into building level operation systems and in conjunction, can be adjusted locally by users that require glare and privacy control.

(3) The proportions (not shape) of cell aperture geometry affects applied strains: The restraining grids that shape individual pneumatic pillows provide an added design opportunity for both systems. Experiments determine that the expansion ratio of a restrained film is relative to the size of the cell aperture measured along the shorter of the two axes, governed by the smaller of the two lengths to affect a film's ability to resist the given load. For a given pressure differential within a single expansion chamber, differences in optical transparency between multiple irregularly shaped apertures can be achieved, as verified in Prototype A1 (F3a \& F8-top left).

\section{DISCUSSION: Summary and Outlook}

\section{Building System Integration}

The paper presents a novel pneumatic actuation strategy for light and privacy control using plasmatreated PDMS film, conceivable for interior partitions, building envelopes, facades and roofs. More technical development is needed to achieve a reliable product to be deployed in a building. For example, the need to protect PDMS film from the elements and from impact (human, environmental, etc.) is common to all applications, the micro-scale surface features of plasma-treated PDMS should be better understood, and little thought has been given so far to understanding the potential thermal characteristics of this system's design. To summarize future steps, a bullet point list of 
research direction is given below.

\section{1. Further research}

- It might be interesting to compare light transmittance results from equally stressed mechanically-pulled and pneumatically-stretched PDMS. However, given the difficulties to develop and maintain a mechanical actuation system at the building scale, it is difficult to justify continuing this line of research.

- Establish rigorous plasma treatment schedules and identify manufacturing impediments to better understand relationships between system performance and cost.

- We also suggest that a complete cost-analysis be prepared to fully understand the potential financial impact of our proposed enclosure system when compared to existing dynamically actuated glazed envelope technologies.

- With reliable production of plasma treated films and a specifically designed set of prototypes having a well defined range of aperture geometries, a meaningful comparison between pneumatic strain simulations and physical transmittance results can occur that will provide the first data sets for developing graphics-based computer simulations.

- With an effective environmental simulation tool in hand predicting transmittance, design cycles can be significantly reduced, simultaneously increasing the predictability of final results.

- Additionally, a comprehensive transmittance simulation model would encourage the exploration of additional user comfort and experience design parameters. For example, PALMS may very well minimize discomforting glare with little reduction to overall transmittance (of diffuse light); with the simulation data in hand, other light analysis tools such as those presented by Wienold and Christoffersen can quickly gain a level of dynamism to become more effective interior environment design tools [32]

- Understanding the mathematical relationships between curvature and level of actuation can be expanded upon. For example, it may be interesting to know where irregularly shaped aperture geometries, such as those exhibited in Prototype A1, produce more subtle light transmittance effects, supporting new design opportunities.

- Research that provides a characterization of the microscale cracking patterns on the doubly curved surface will provide insight into canceling effect between touching film surfaces (System B). Establishing new guidelines can become design opportunities for more dynamic expansion chambers and aperture arrays.

- More research is needed to quantify the affect of PALMS' $s$ when covering a significant portion of the facade. The pneumatic element can potentially serve to reduce thermal conductivity of the building envelope and additional which may reduce its operational energy consumption.

- Most existing data is referencing untreated PDMS [20]. It is important to formally establish the life cycle of the plasma treated PDMS in this system to establish its resistance to UV light and ability to withstand continuous changes in applied strain when oxidized.

- Measuring light transmittance (beyond normal incidence transmission) would benefit from the use of an integrating sphere to examine the contribution of scattered and diffused light through a PDMS sample. A full analysis and quantification of the light scattering material properties would complement this study and provide a broader understanding of the PALMS mechanisms used to affect a user's environment, and provide more data for graphics simulation. 
- Also, light transmittance data collection should encompass a larger wavelength range including the near-infrared response and follow EN 410 (and ISO 9050:2003) guidelines to determine PALMS's luminous solar characteristics.

\section{Figure 15: ETFE Pillow encapsulation}

\subsection{Integrating PALMS into existing envelope systems}

When deploying a system within a typical glazed window section, accessibility to the pneumatic components through removable glass panels is likely an advantage. Protection could be achieved by designing the pneumatic layer located within a glazing system or inside a pneumatic ETFE pillow; here the light control system can simply connect to an already existing infrastructure of air pumps and distribution ducts. Air pressure lines can be small, incorporated into the profiles of a typical assembly frame.

The required pumps and compressors for both Systems A \& B can be easily integrated into an HVAC system in a cost-effective way. Pneumatic facades made from ETFE and other films are particularly promising because the air handling equipment and air supply networks they require are already in place. However, the pressures required to activate System A are significantly higher than those found in existing ETFE facades, where typically $1.4 \mathrm{kPa}$ is needed in case of high wind loads [19]. In addition, normal expansion ratios for ETFE envelope systems (5-6:1, ' pillow' :aperture radii) are much higher than what PALMS requires (1:1' pillow' aperture radii). System A deploys positive pressure, and System B relies on negative pressure. Both can produce strained PDMS sheets with similar curvatures, requiring an approximate 2-2.5:1 expansion ratio at $10.3 \mathrm{kPa}$ of pressure for positive expansion and $\langle 177.8 \mathrm{~mm} \mathrm{Hg}(<-23.7 \mathrm{kPa})$ for negative pressure. However, System A expands with positive pressure, making System B better suited for constrained spatial conditions because it retains constant sectional depth. For these reasons, the authors propose the System B solution as an interlayer for ETFE pillow facades (F15), replacing the now frequently placed central ETFE film that is often used to reduce thermal conductivity; the double layer PDMS system would do so more efficiently since an additional vacuum layer is created inside the PALMS chamber. Therefore, it seems promising to improve an existing pneumatic infrastructure with a limited investment by adding privacy control and exploiting potential thermal advantages. More research is needed to

characterize the behavior of such composite, multi-scalar pneumatic facades, and to design them for optimal performance.

\section{3. Acknowledgements:}

Agnello, Elyse; Blough, Tom; Hess, Kelley; King, Jonathan; Overvelde, Johannes; Singh, Malika; Weaver, James.

\section{References}

[1] Z. Drozdowski, S. Gupta, Adaptive fritting as case exploration for adaptivity in architecture, in: ACADIA 09:ReForm() : Building a Better To- morrow : Proceedings of the 29th Annual Conference of the Association for Computer Aided Design in Architecture (ACADIA), 2009. 
[2] F. Eyl, Aperture - studio thegreeneyl (April 2017) [cited 23 April, 2017].

URL http://www. thegreeneyl. com/aperture

[3] F. L 'opez Jim enez, S. Kumar, P. M. Reis, Soft color composites with tunable optical transmittance, Advanced Optical Materials 4 (4) (2016) 620-626. doi:10.1002/adom. 201500617. URL http://dx. doi.org/10. 1002/adom. 201500617

[4] A. B. Ucar, 0. D. Velev, Microfluidic elastomer composites with switchable vis-IR transmittance, Soft Matter 8 (2012) 11232-11235. doi:10.1039/ C2SM26635K. URL http://dx. doi. org/10. 1039/C2SM26635K

[5] R. Baetens, B. P. Jelle, A. Gustavsen, Properties, requirements and possibilities of smart windows for dynamic daylight and so- lar energy control in buildings: A state-of-the-art review, So- lar Energy Materials and Solar Ce11s 94 (2) (2010) 87 - 105.

doi:https://doi. org/10. 1016/j. solmat. 2009. 08. 021.

URL http://www. sciencedirect. com/science/article/pii/ S0927024809002992

[6] S. E. Selkowitz, C. M. Lampert, Application of Large-Area Chromogenics to Architectural Glazings, 1990.

[7] R. Yin, P. Xu, P. Shen, Case study: Energy savings from solar window film in two commercial buildings in shanghai, Energy and Buildings 45 (Supplement C) (2012) 132 - 140.

doi:https://doi. org/10. 1016/j. enbuild. 2011. 10. 062.

URL http://www. sciencedirect.com/science/article/pii/ S0378778811005251

[8] N. L. Sbar, L. Podbelski, H. M. Yang, B. Pease, Electrochromic dynamic windows for office buildings, International Journal of Sustainable Built Environment 1 (1) (2012) 125 - 139.

doi:https: //doi. org/10. 1016/j. i jsbe.2012.09.001. URL

http://www. sciencedirect.com/science/article/pii/ S2212609012000118

[9] C. M. Lampert, Smart switchable glazing for solar energy and daylight control, Solar Energy Materials and Solar Cells 52 (3) (1998) 207 - 221. doi:https://doi.org/10. 1016/S09270248 (97) 00279-1.

URL http://www. sciencedirect.com/science/article/pii/ S0927024897002791

[10] R. Vergaz, J. -M. Sa 'nchez-Pena, D. Barrios, C. V'azquez, P. Contreras- Lallana, Modelling and electro-optical testing of suspended particle devices, Solar Energy Materials and Solar Cells 92 (11) (2008) 1483 - 1487. doi:https://doi.org/10. 1016/j. solmat. 2008. 06. 018. URL http://www. sciencedirect. com/science/article/pii/ S0927024808002146

[11] E. E. Glass, Electric privacy glass [cited Feburary 5, 2015]. URL http://www. eclipseelectricglass. co. uk/

[12] F. Goia, M. Perino, V. Serra, Improving thermal comfort conditions by means of pcm glazing systems, Energy and Buildings 60 (2013) $442-452$. doi:https://doi. org/10. 1016/j. enbuild. 2013.01.029.

URL http://www. sciencedirect.com/science/article/pii/ S0378778813000601 
[13] L. Giovannini, F. Goia, V. R. L. Verso, V. Serra, Phase change materials in glazing: Implications on light distribution and visual comfort. preliminary results, Energy Procedia 111 (2017) 357 - 366, 8th International Conference on Sustainability in Energy and Buildings, SEB16, 11-13 September 2016, Turin, Italy. doi:https://doi. org/10. 1016/j. egypro. 2017. 03. 197. URL http://www. sciencedirect. com/science/article/pii/ S1876610217302278

[14] D. Li, T. Ma, C. Liu, Y. Zheng, Z. Wang, X. Liu, Thermal performance of a pcm-filled double glazing unit with different optical properties of phase change material, Energy and Buildings 119 (2016) 143 - 152. doi:https://doi. org/10. 1016/j. enbuild. 2016. 03.036. URL http://www. sciencedirect. com/science/article/pii/ S0378778816301736

[15] Vector-Foiltec, Solar control [cited 15 April, 2017]. URL http://www. vectorfoiltec. com/texlon-etfe-system/ solar-control/

[16] E. Ruiz-Geli, Enric ruiz-geli - projects: Built projects - media-ict [cited 26 September, 2016].

URL http://www. ruiz-geli. com/projects/built/media-tic

[17] S. Yang, K. Khare, P. -C. Lin, Harnessing surface wrinkle patterns in soft matter, Advanced Functional Materials 20 (16) (2010) 2550-2564. doi: 10.1002/adfm. 201000034.

URL http://dx. doi.org/10. 1002/adfm. 201000034

[18] P. Kim, Y. Hu, J. Alvarenga, M. Kolle, Z. Suo, J. Aizenberg, Rational design of mechanoresponsive optical materials by fine tuning the evolution of strain-dependent wrinkling patterns, Advanced Optical Materials 1 (5) (2013) 381-388. doi:10. 1002/adom. 201300034. URL http://dx. doi.org/10. 1002/adom. 201300034

[19] D. Park, P. Kim, J. Alvarenga, K. Jin, J. Aizenberg, M. Bechthold, Dynamic daylight control system implementing thin cast arrays of polydimethylsiloxane-based millimeter-scale transparent louvers, Building and Environment 82 (Supplement C) (2014) 87 - 96. doi:https://doi. org/10. 1016/j. buildenv. 2014.07.016. URL http://www. sciencedirect.com/science/article/pii/ S0360132314002406

[20] Polymer data handbook, 2nd ed., Journal of the American Chemical Society 131 (44) (2009) 16330-16330, pMID: 19764753. arXiv:https://doi.org/10.1021/ja907879q, doi:10. 1021/ja907879q. URL https://doi.org/10. 1021/ja907879q

[21] C. Wiesmeier, I. Haedrich, K. -A. Weiss, I. Duerr, Overview of pv module encapsulation materials 19 (2013) 85-92.

[22] Wacker Chemie AG. Solid and Liquid Silicone Rubber Material and Processing Guidlines. München, Germany: Wacker Chemie AG. Pg (84). Accessed April 8, 2018. https://www. wacker. com/cms/media/publications/downloads/6709_EN.pdf.

[23] P. Jagdale, S. Salimpour, M. Hujjatul Islam, F. Cuttica, F. Robles- Hern 'andez, A. Tagliaferro, A. Frache, Flame retardant effect of nano fillers on polydimethylsiloxane composites 18 (2018) 1468-1473. 
[24] C. F. Guo, Q. Liu, G. Wang, Y. Wang, Z. Shi, Z. Suo, C. -W. Chu, Z. Ren, Fatigue-free, superstretchable, transparent, and biocompat-ible metal electrodes, Proceedings of the National Academy of Sciences 112 (40) (2015) 12332-12337. arXiv:http://www. pnas. org/content/112/ 40/12332. full. pdf, doi:10. 1073/pnas. 1516873112. URL http://www. pnas. org/content/112/40/12332

[25] K-mac Plastics, ETFE Tefzel Sheets and Film [cited 5 April, 2018]. URL http://www. k-macplastics. com/etfe-sheets-film. htm

[26] K-mac Plastics, Translucent Silicone Sheets [cited 5 April, 2018]. URL http://k-macplastics. com/silicone-semi-clear-sheets. htm

[27] "Halar High Clarity ECTFE Transparent Films for Architectural Applications." Solvay Polymers. Accessed January 23, 2018. https://www. solvay. jp/ja/binaries/Halar-High-Clarity-ECTFETransparent-Films-for-Architectural-Applications_EN-240768. pdf.

[28] I. Johnston, D. Mccluskey, C. Tan, M. Tracey, Mechanical characterization of bulk sylgard 184 for microfluidics and microengineering 24 (2014) 035017.

[29] Z. Huang, W. Hong, Z. Suo, Nonlinear analyses of wrinkles in a film bonded to a compliant substrate, Journal of the Mechan- ics and Physics of Solids 53 (9) (2005) 2101 - 2118. doi:https: //doi. org/10. 1016/j. jmps. 2005. 03. 007.

URL http://www. sciencedirect. com/science/article/pii/ S0022509605000700

[30] HP 3D Scan, HP 3D Structured Light Scanner Pro S3 [cited 4 April, 2018]. URL http://www8. hp. com/us/en/campaign/3Dscanner/overview. html\#products

[31] N. Bowden, S. Brittain, A. G. Evans, J. W. Hutchinson, G. M. Whitesides, Spontaneous formation of ordered structures in thin films of metals supported on an elastomeric polymer, Nature 393 (6681) (1998) 146-149.

URL http://dx. doi.org/10. 1038/30193

[32] J. Wienold, J. Christoffersen, Evaluation methods and development of a new glare prediction model for daylight environments with the use of ccd cameras, Energy and Buildings 38 (7) (2006) 743 - 757, special Issue on Daylighting Buildings. doi:https://doi.org/10.1016/j. enbuild. 2006. 03. 017. URL http://www. sciencedirect.com/science/article/pii/ S0378778806000715 


\section{Figure Captions}

Figure 1: System A, Positive Pressure
(a) Prototype A2: at rest
(b) Prototype A2: actuated

Figure 2: Micro-scale surface cracks on plasma-treated PDMS film. Left: Photographs of micro- scale surface features of pneumatically unstrained and strained plasma-treated film. Top Left: sample at rest; Bottom Left: actuated sample. Right: Illustration of pneumatically strained plasma treated PDMS diagramming light scattering optical effect.

Figure 3: System Prototypes
(a) System A: Positive Pressure
(b) System B: Negative Pressure

Figure 4: System A section. Above: System A, at rest. Below: System A, actuated

Figure 5: System B section. Above: System B, at rest. Below: System B, actuated

Figure 6: System A typical assembly

Figure 7: Constraining grid array

Figure 8: System A Prototypes. Clockwise from top left: A1, A2, A3, A4

Figure 9: Prototype B2, Negative Pressure

a) Prototype B2 at rest (above) and actuated (below) (b) The cell-width and cell-depth ratio is ideally 1:1

Figure 10: Spectrometer set-up diagram

Figure 11: Prototype A5, $10 \mathrm{~cm} 2$ aperture

Figure 12: Fully Actuated System: Actual to Simulation Comparison
(a) Photograph of light transmission of an actual sample
(b) Strain simulation, showing max in-plane strain.

Figure 13: Aperture width to radii of curvature: Left column: elongated aperture. Right column: equal proportioned aperture Aperture proportions largely determine the degree to which uniform strains develop in the inflated condition. Ideal conditions are present in a circular opening, whereas elongated apertures generally expand to a max height dictated by the minor dimension. This reduces force in one dimension of the pneumatically applied bi-axial strain for a given pressure thereby reducing effective change in opacity. 
Figure 14: Early shape experiments at $117.2 \mathrm{kPa}$ Relationship between aperture proportion and opacity. Above: Long-narrow proportion. Below: Near equal length-width proportion.

\section{Figure 15: ETFE Pillow encapsulation}

Tables

\begin{tabular}{|c|c|c|}
\hline Material & ETFE & PDMS \\
\hline Tensile Strength $[\mathrm{MPa}]$ & $38-48$ & 5.5 \\
\hline Elongation $[\%]$ & $100-350$ & 250 \\
\hline Thermal Conductivity $[\mathrm{W} / \mathrm{m} \cdot \mathrm{K}]$ & 0.238 & 0.21 \\
\hline Total Transmittance & $93.8+/-0.1$ & $97.00 \%$ \\
\hline Haze & $9.2+/-0.8$ & \\
\hline Cost (film sheets) & $\begin{array}{c}0.127 \mathrm{~mm} \text { thickness: \$327USD } / \mathrm{m}^{2} \\
{[25]}\end{array}$ & $\begin{array}{l}0.508 \mathrm{~mm} \text { thickness: \$258USD / } \\
\qquad \mathrm{m}^{2}[26]\end{array}$ \\
\hline
\end{tabular}

Table 1: Material property comparison between ETFE and PDMS films.

\begin{tabular}{|c|c|c|c|c|}
\hline Sample State & Position & $\begin{array}{c}\text { \% Transmittance } \\
(\mathbf{4 0 0 - 9 0 0 n m ~ m e a n ~ v a l u e ) ~}\end{array}$ & $\begin{array}{c}\text { Min In-Plane } \\
\text { Logarithmic Strain }\end{array}$ & $\begin{array}{c}\text { Max In-Plane } \\
\text { Logarithmic Strain }\end{array}$ \\
\hline Actuated & $(-3,3)$ & 18.8 & $2.40 \mathrm{E}-01$ & $3.20 \mathrm{E}-01$ \\
\hline Actuated & $(-3,0)$ & 19.1 & $3.20 \mathrm{E}-01$ & $3.90 \mathrm{E}-01$ \\
\hline Actuated & $(0,3)$ & 21.6 & $3.20 \mathrm{E}-01$ & $3.90 \mathrm{E}-01$ \\
\hline Actuated & $(0,0)$ & 18.1 & $4.10 \mathrm{E}-01$ & $4.20 \mathrm{E}-01$ \\
\hline Relaxed & $(-3,3)$ & 71.7 & & \\
\hline Relaxed & $(-3,0)$ & 71.5 & & \\
\hline Relaxed & $(0,3)$ & 69.1 & & \\
\hline Relaxed & $(0,0)$ & 67.5 & & \\
\hline
\end{tabular}

Table 1 2: Transmittance measurements of A5 when fully actuated (see F12a-b). Note the change in a viewers ability to fully recognize shapes (in the case of Fig 12a, vertical lines) through a PDMS sample at rest and while fully actuated. 
Click here to download high resolution image

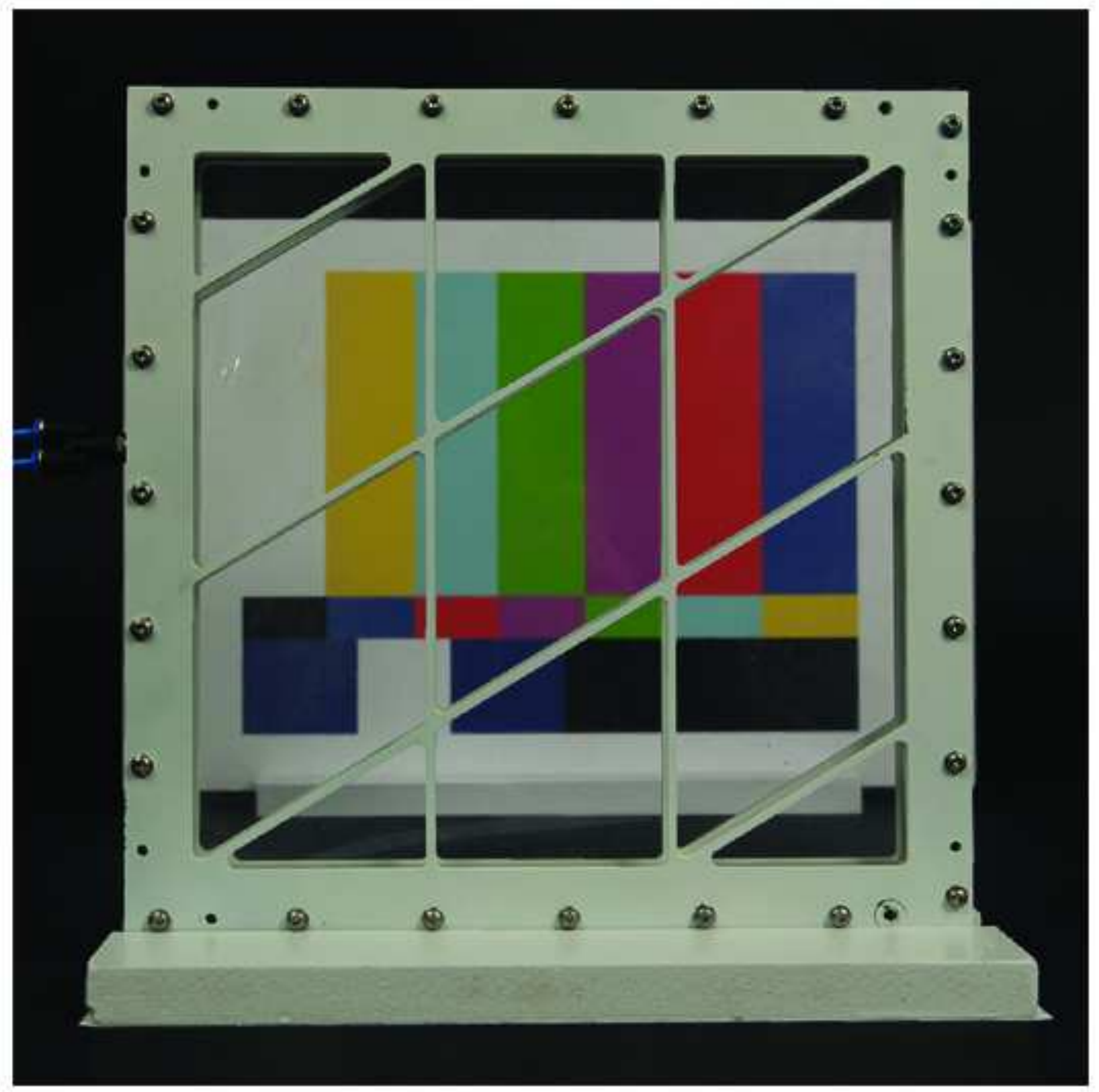




\section{Figure 1b}

Click here to download high resolution image

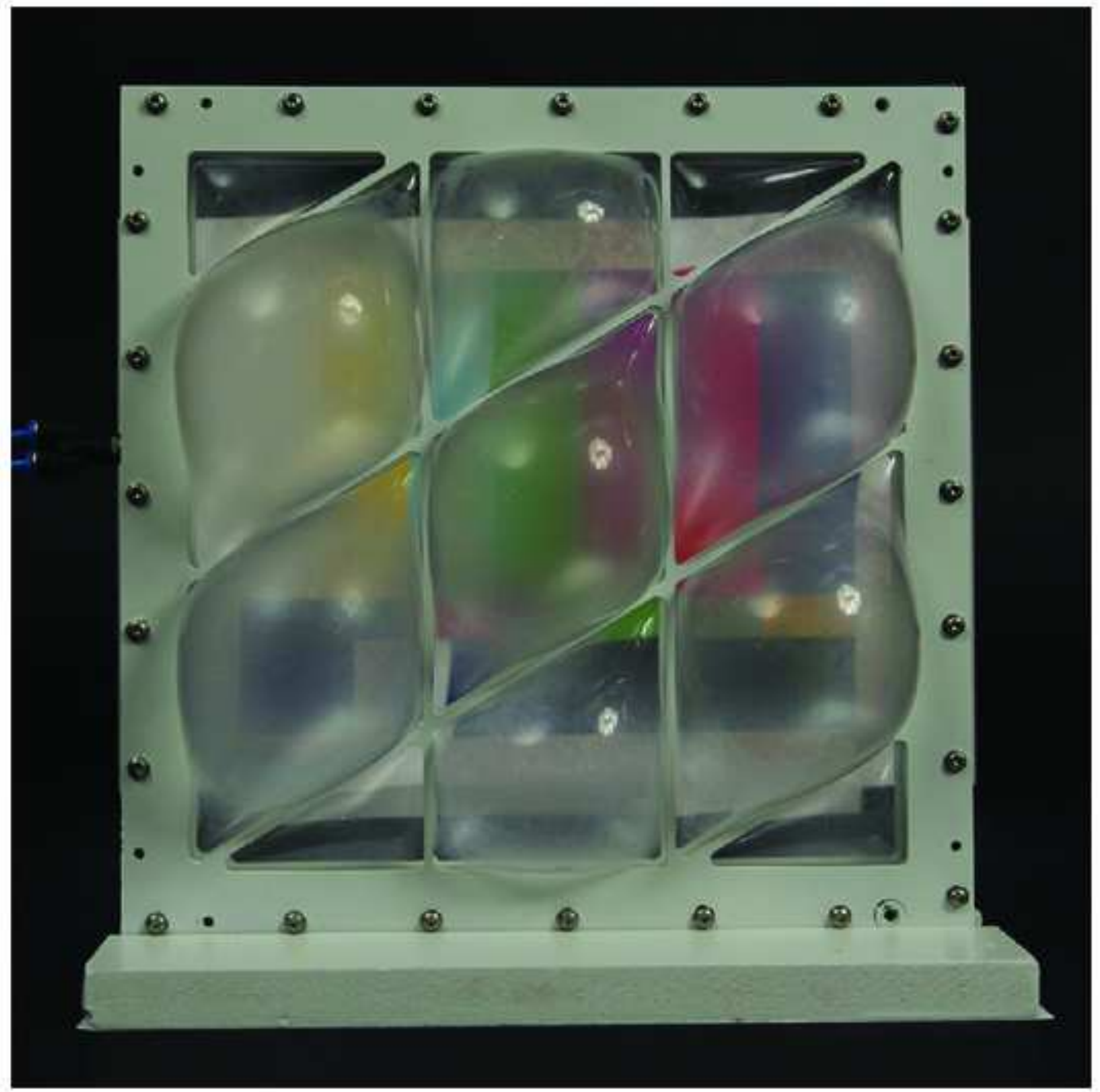




\section{gure 2}

Click here to download high resolution image

Interior Surface 20X Magnification

No Strain

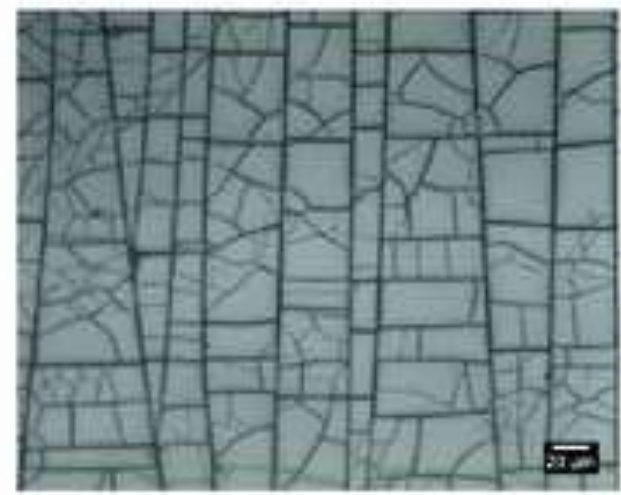

Strain

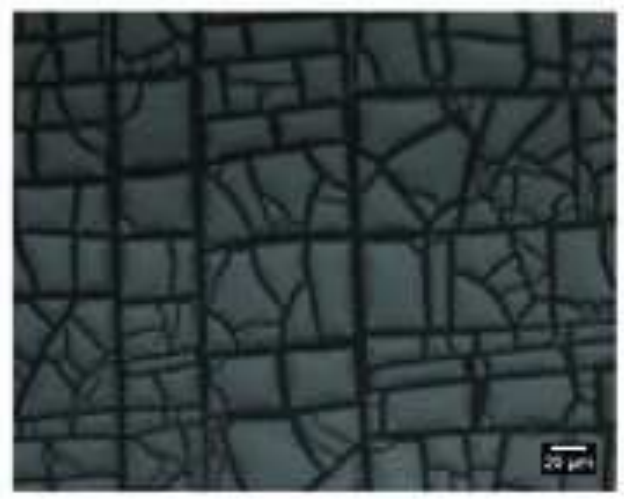

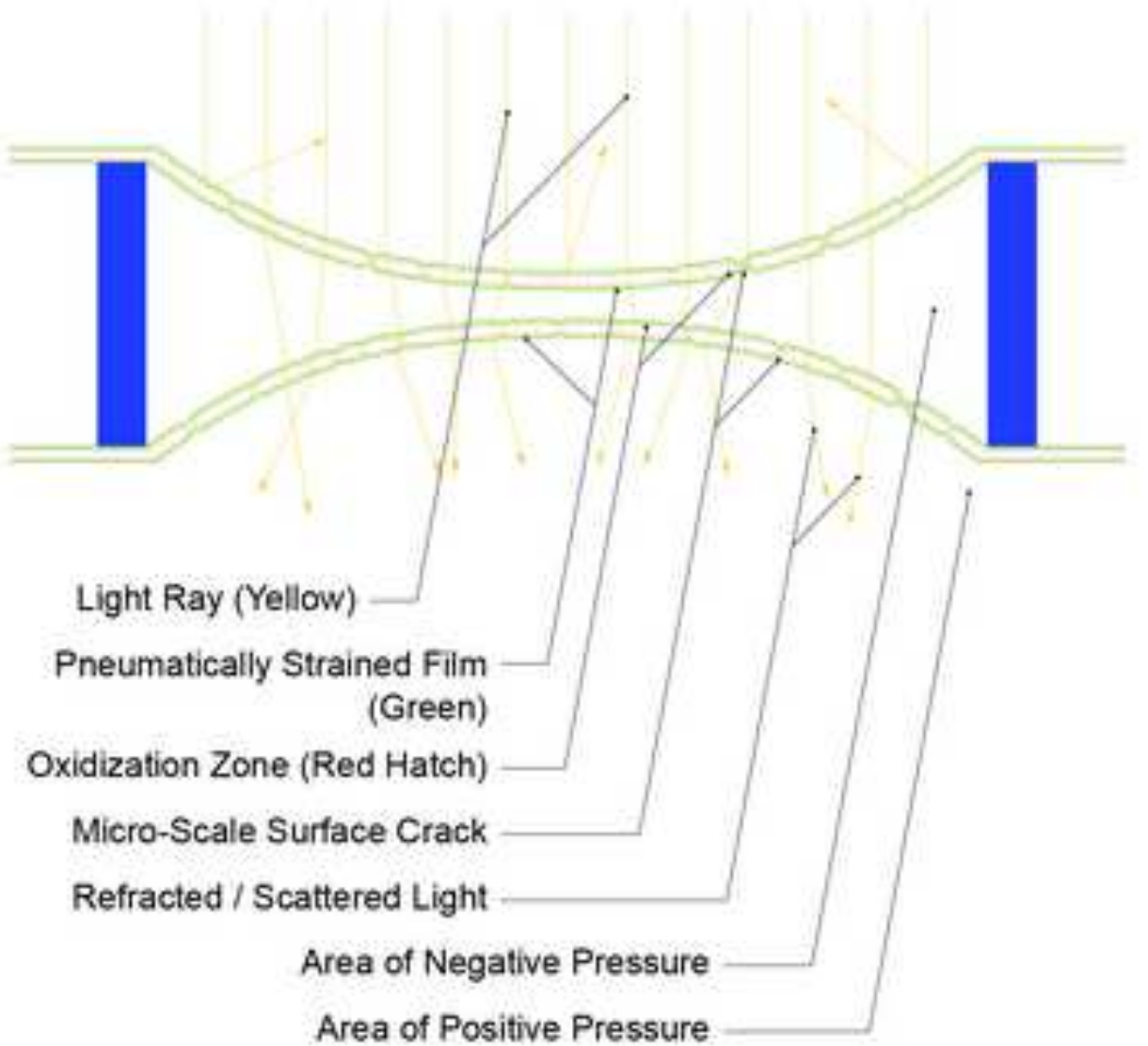


Click here to download high resolution image

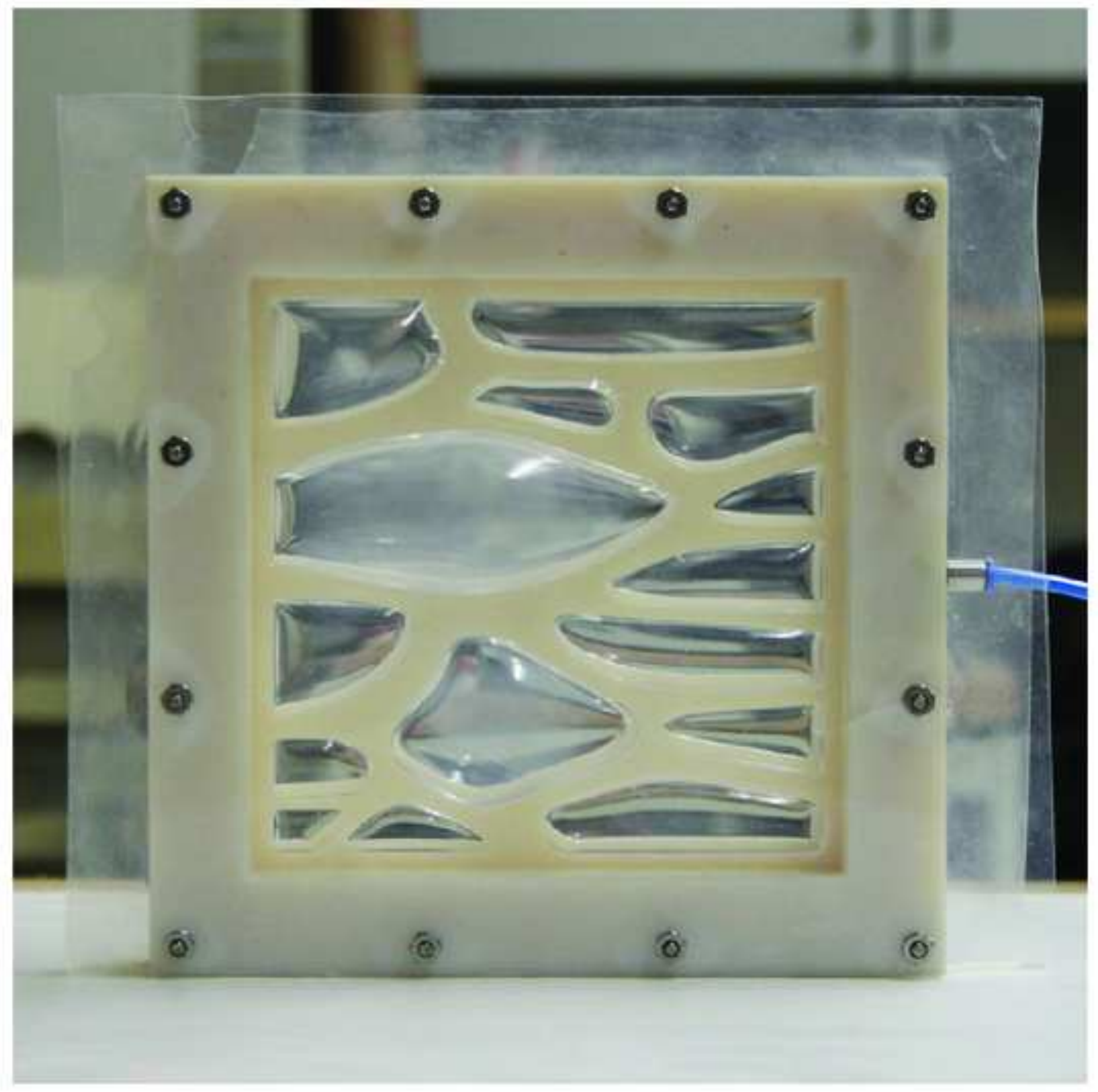


Figure $3 b$
Click here to download high resolution image

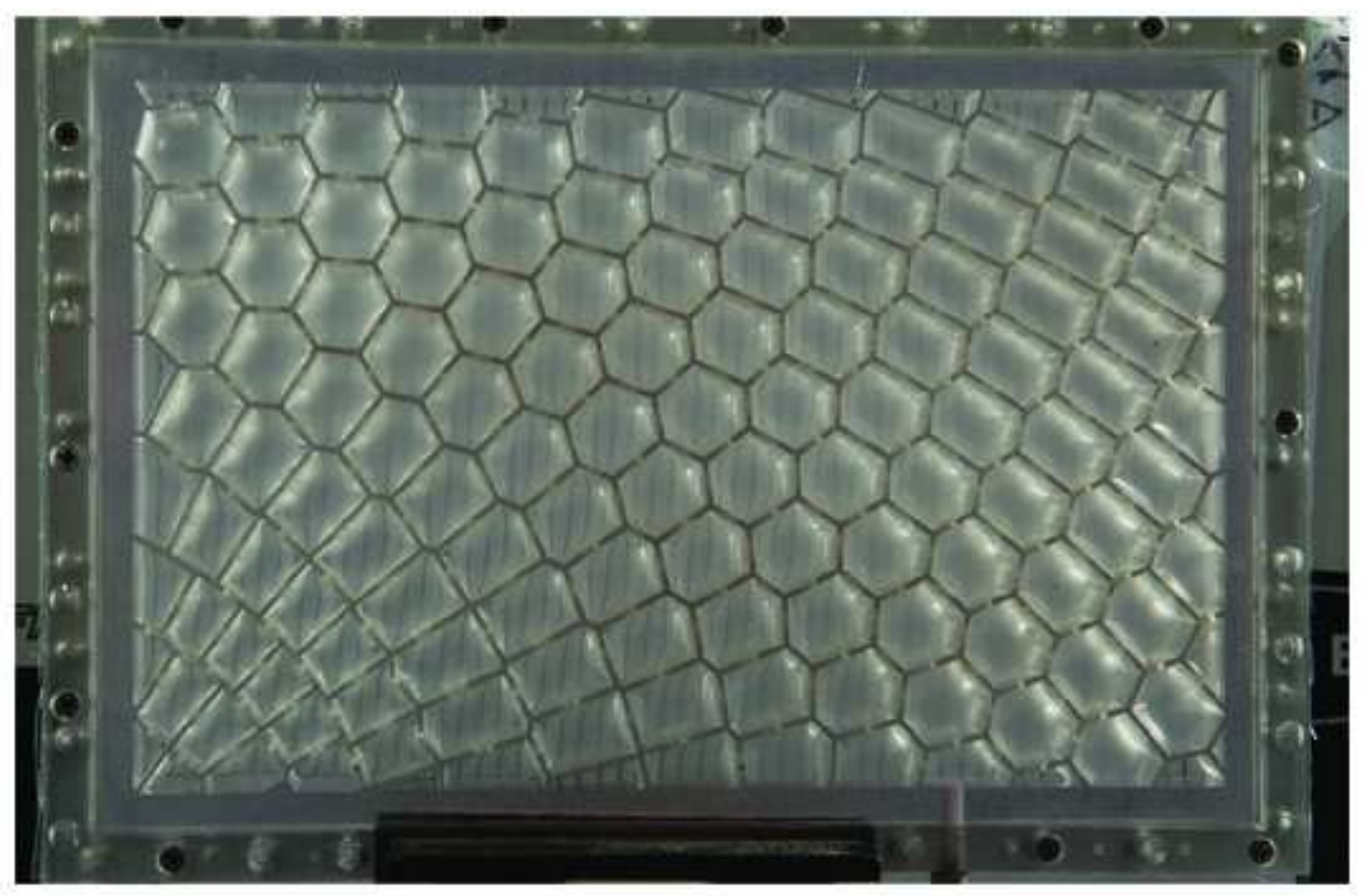




\section{igure 4}

Click here to download high resolution image

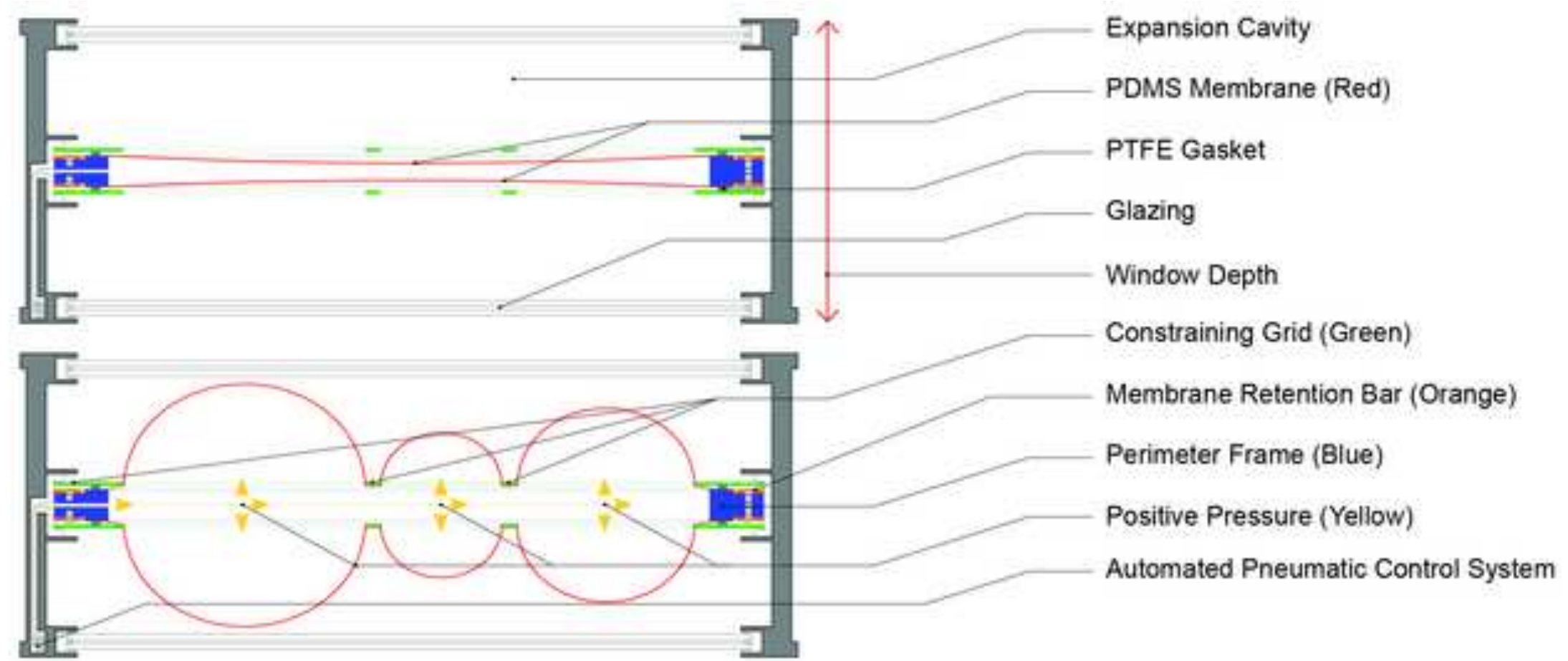




\section{igure 5}

Click here to download high resolution image

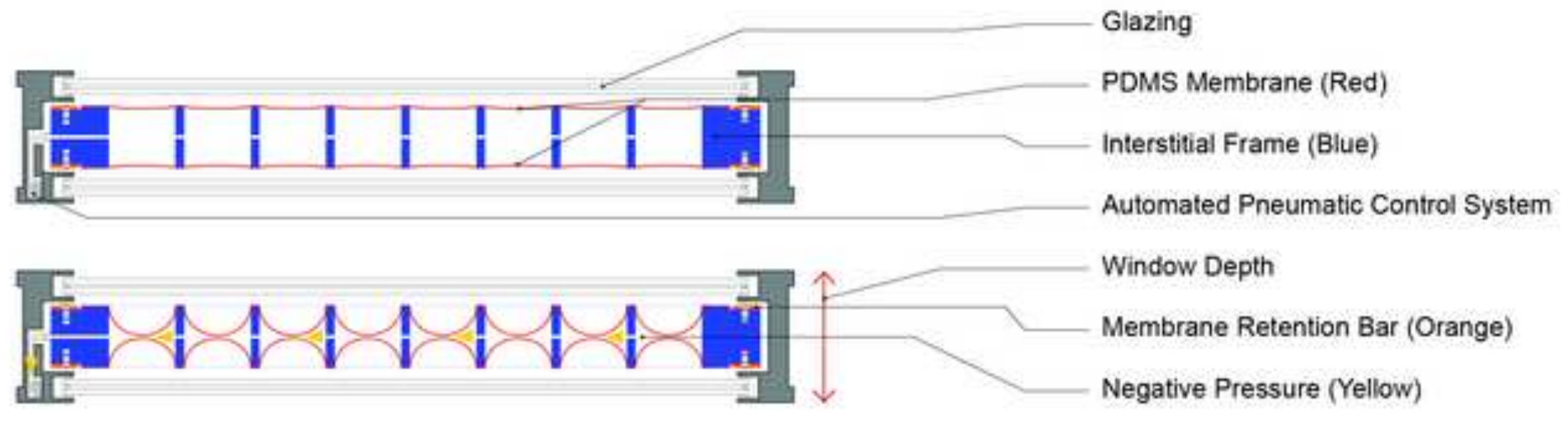


Constraining Grid

PTFE Gasket

Membrane

Retention Bar

Plasma Treated

PDMS Membrane

Aluminum

Perimeter Frame

Plasma Treated PDMS Membrane

Membrane

Retention Bar

PTFE Gasket

Constraining Grid 
Figure 7

Click here to download high resolution image

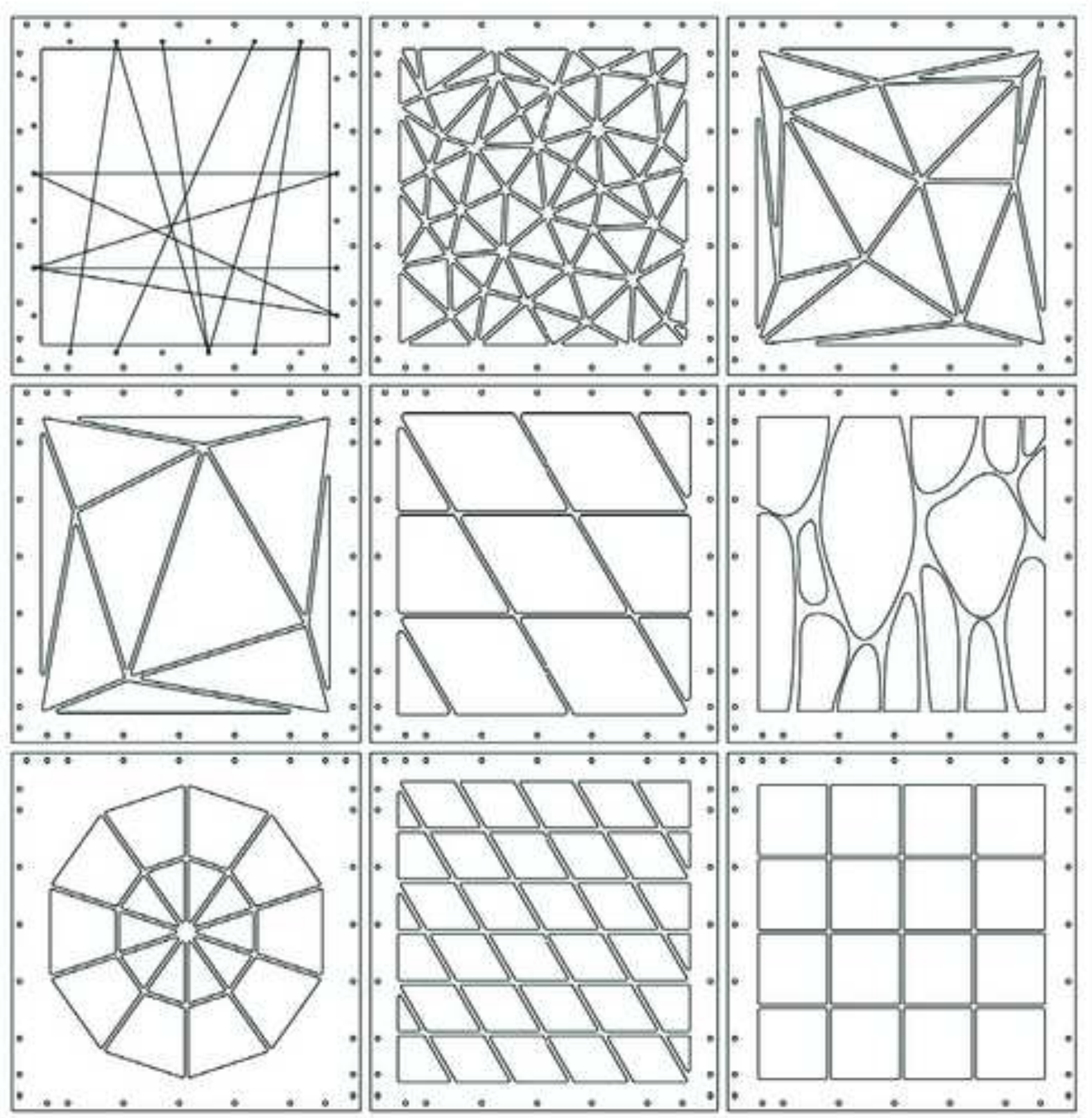


Click here to download high resolution image
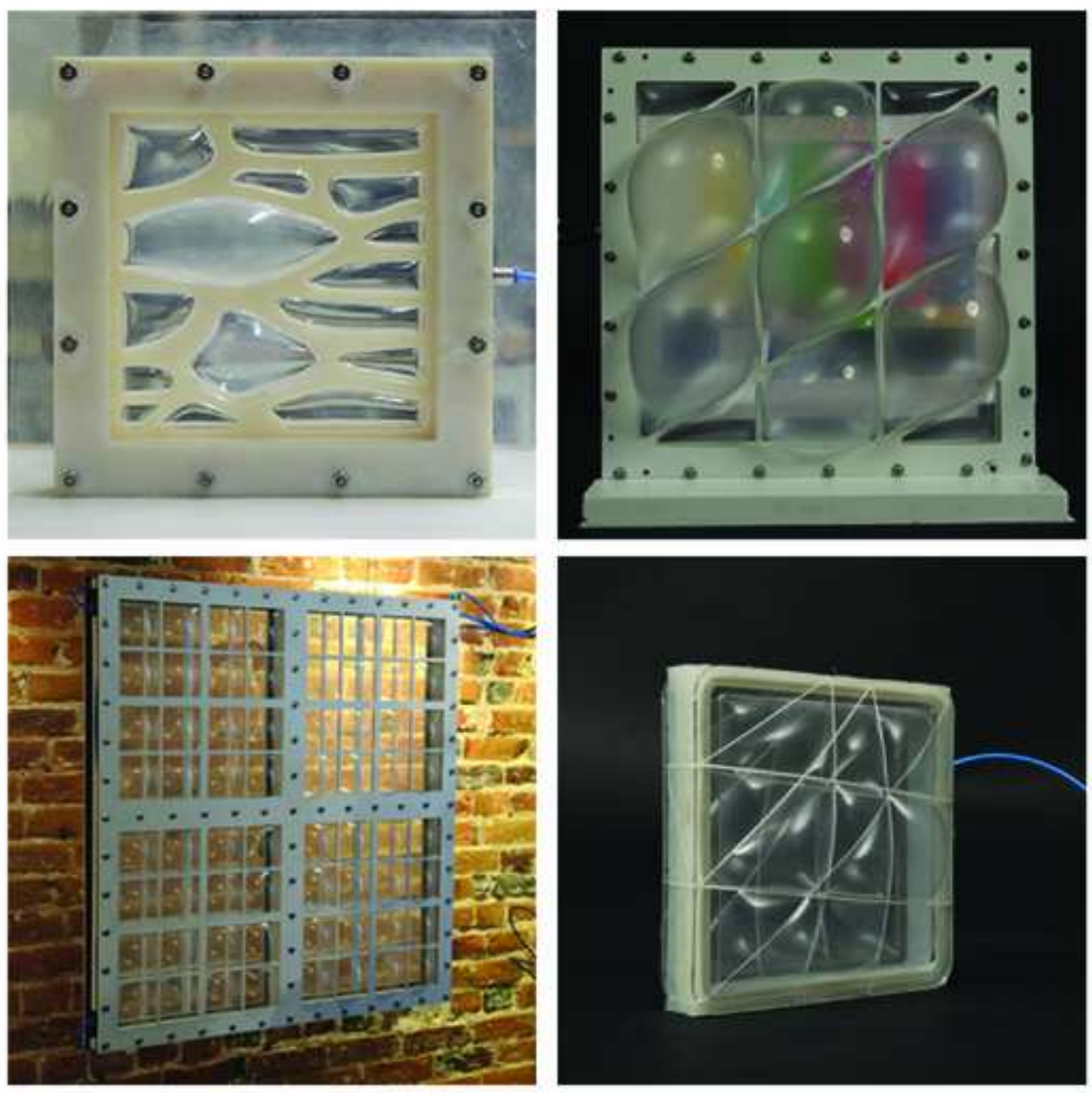


\section{Figure 9a}

Click here to download high resolution image
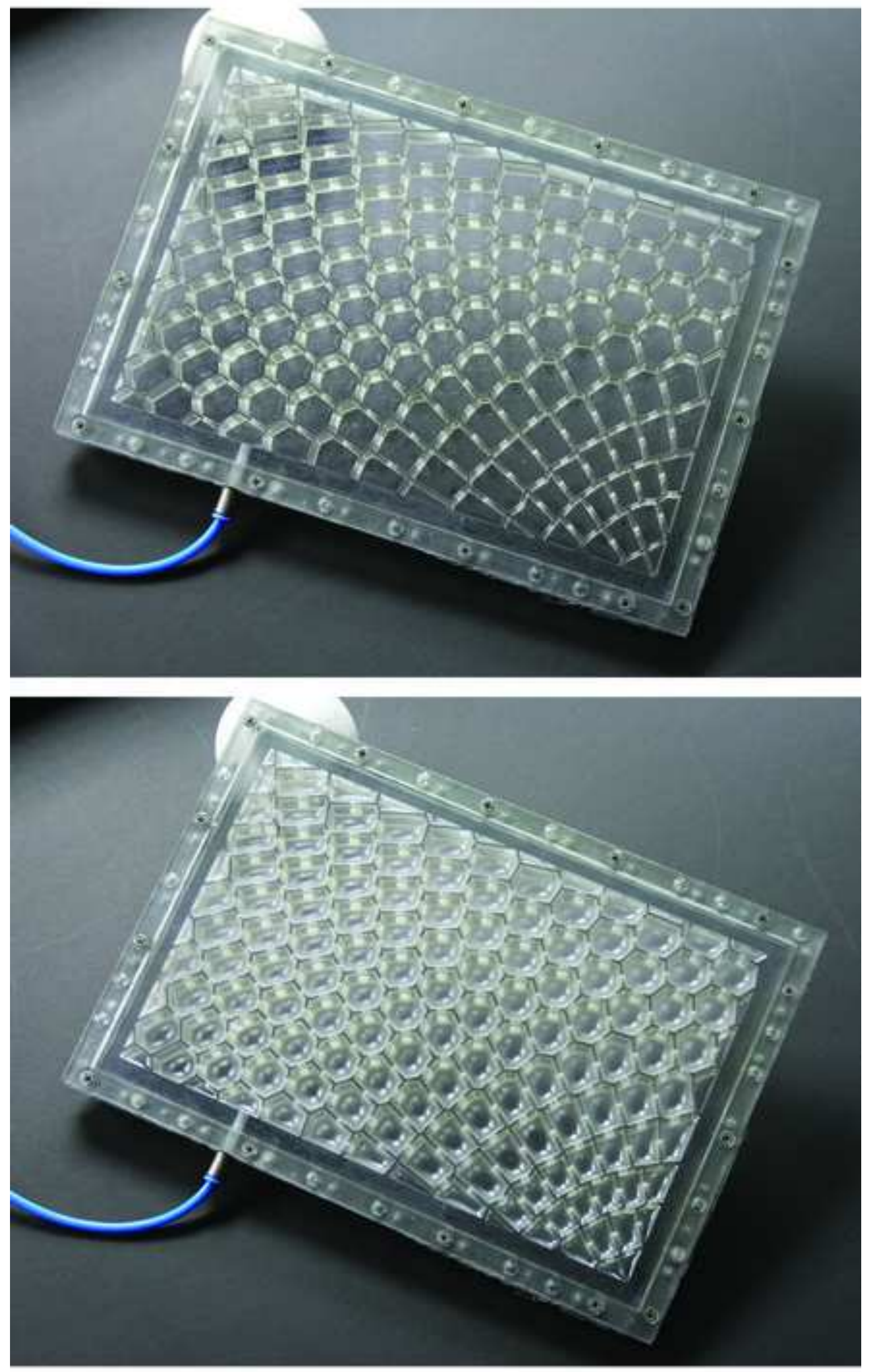


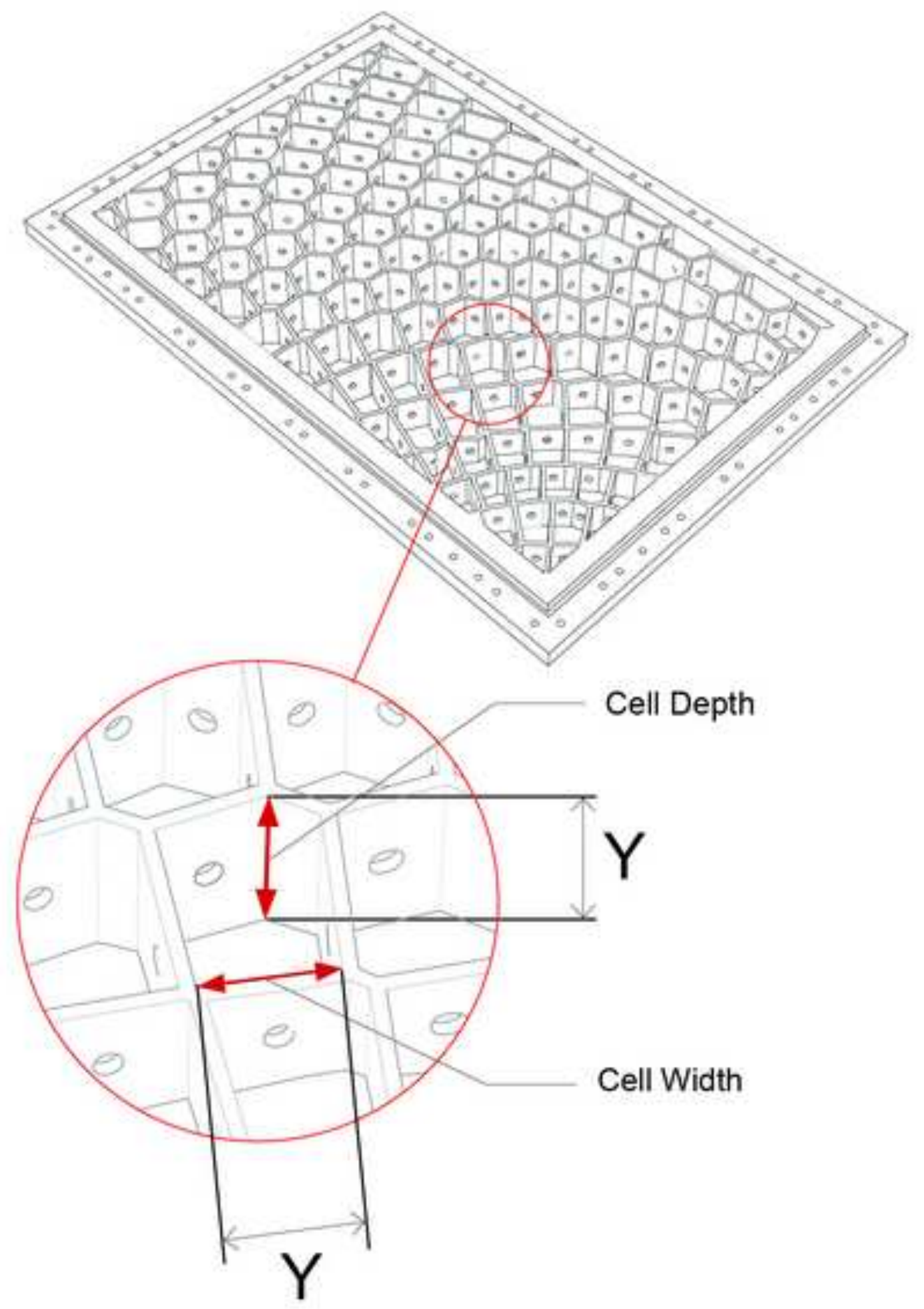




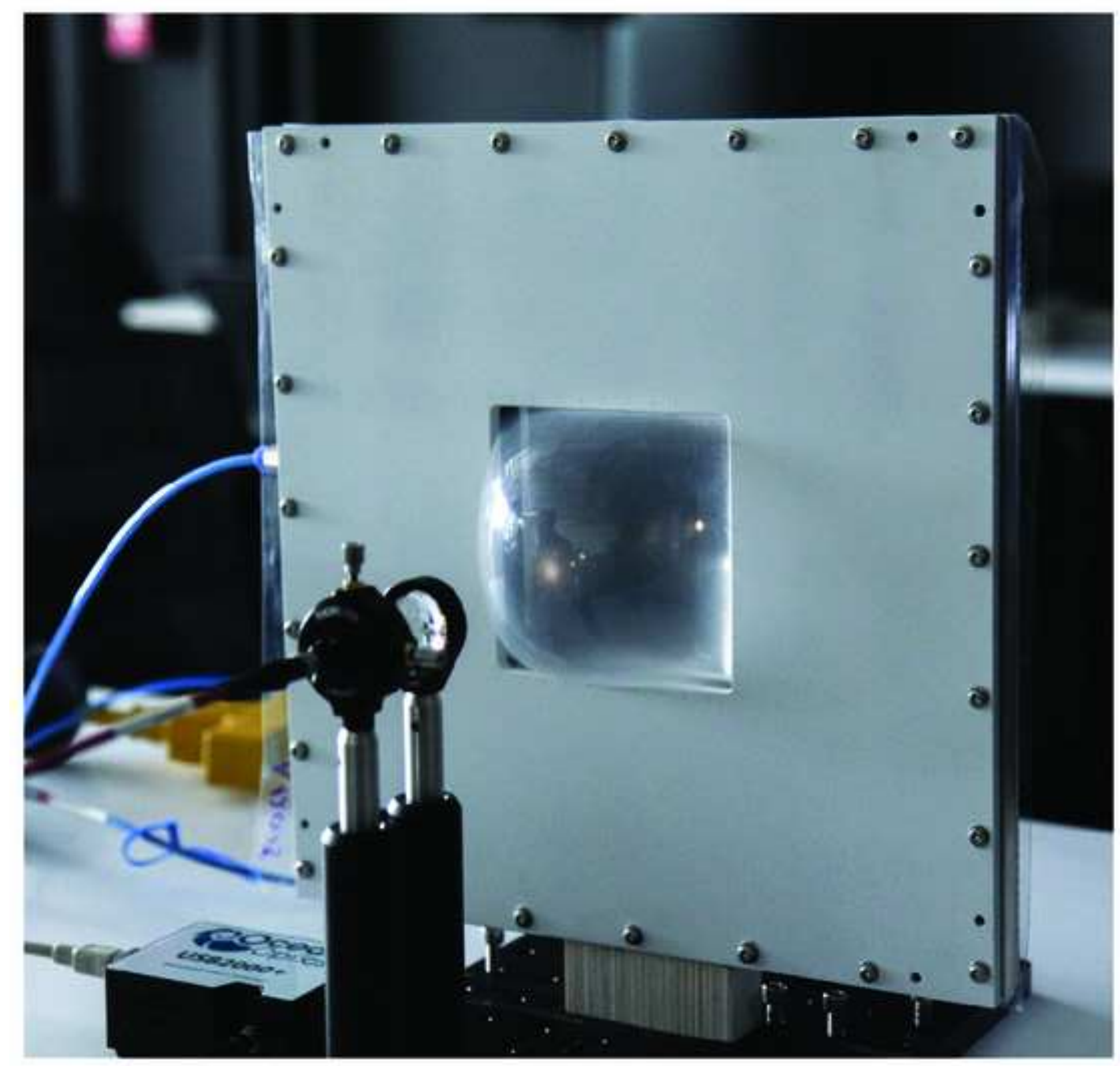

Figure 10
Click here to download high resolution image 
Click here to download high resolution image

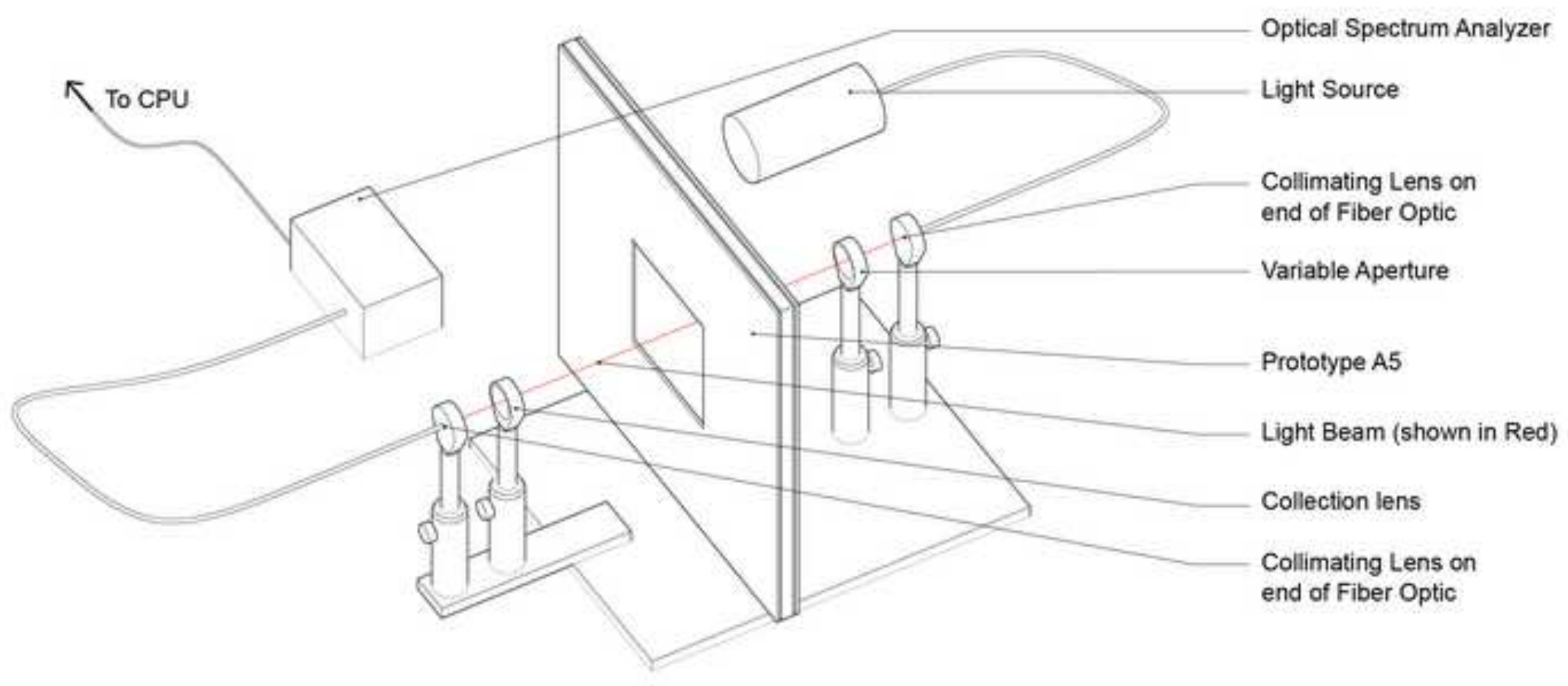




$$
\begin{array}{lc}
(-3,3) & (0,3) \\
(-3,0) & (0,0)
\end{array}
$$


Figure 12

Click here to download high resolution image

\begin{tabular}{|l|}
\hline LE, Max. In-Plane Principal \\
SNEG, (fraction =-1.0) \\
(Avg: $75 \%$ ) \\
$\begin{array}{r}+4.172 \mathrm{e}-01 \\
+3.959 \mathrm{e}-01 \\
+3.747 \mathrm{e}-01 \\
+3.535 \mathrm{e}-01 \\
+3.322 \mathrm{e}-01 \\
+3.110 \mathrm{e}-01 \\
+2.897 \mathrm{e}-01 \\
+2.685 \mathrm{e}-01 \\
+2.473 \mathrm{e}-01 \\
+2.260 \mathrm{e}-01 \\
+2.048 \mathrm{e}-01 \\
+1.835 \mathrm{e}-01 \\
+1.623 \mathrm{e}-01 \\
+1.411 \mathrm{e}-01 \\
+1.198 \mathrm{e}-01 \\
+9.857 \mathrm{e}-02 \\
+7.733 \mathrm{e}-02 \\
+5.609 \mathrm{e}-02 \\
+3.485 \mathrm{e}-02 \\
+1.361 \mathrm{e}-02 \\
-7.627 \mathrm{e}-03\end{array}$ \\
\hline
\end{tabular}

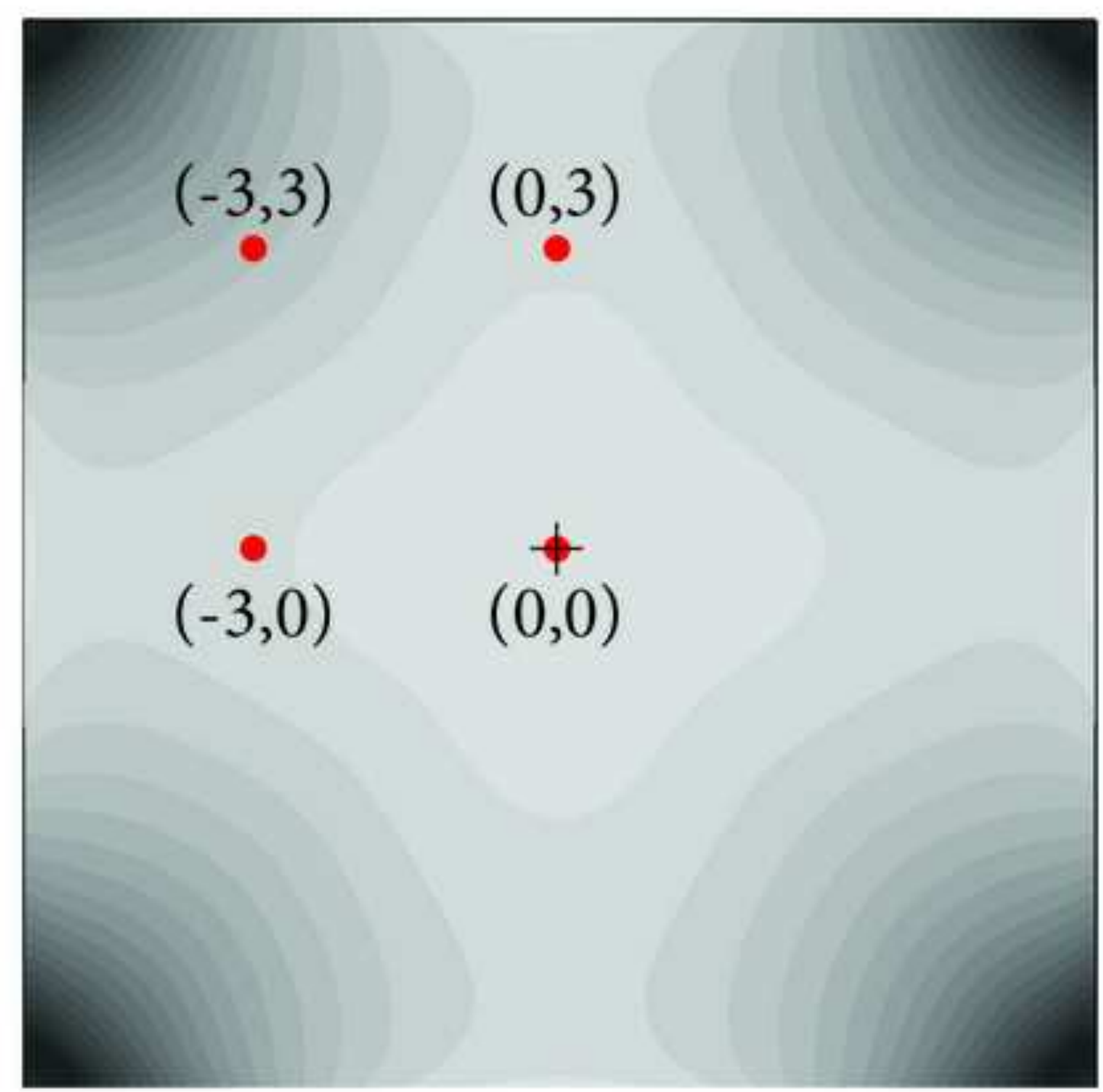


Figure 13
Click here to download high resolution image
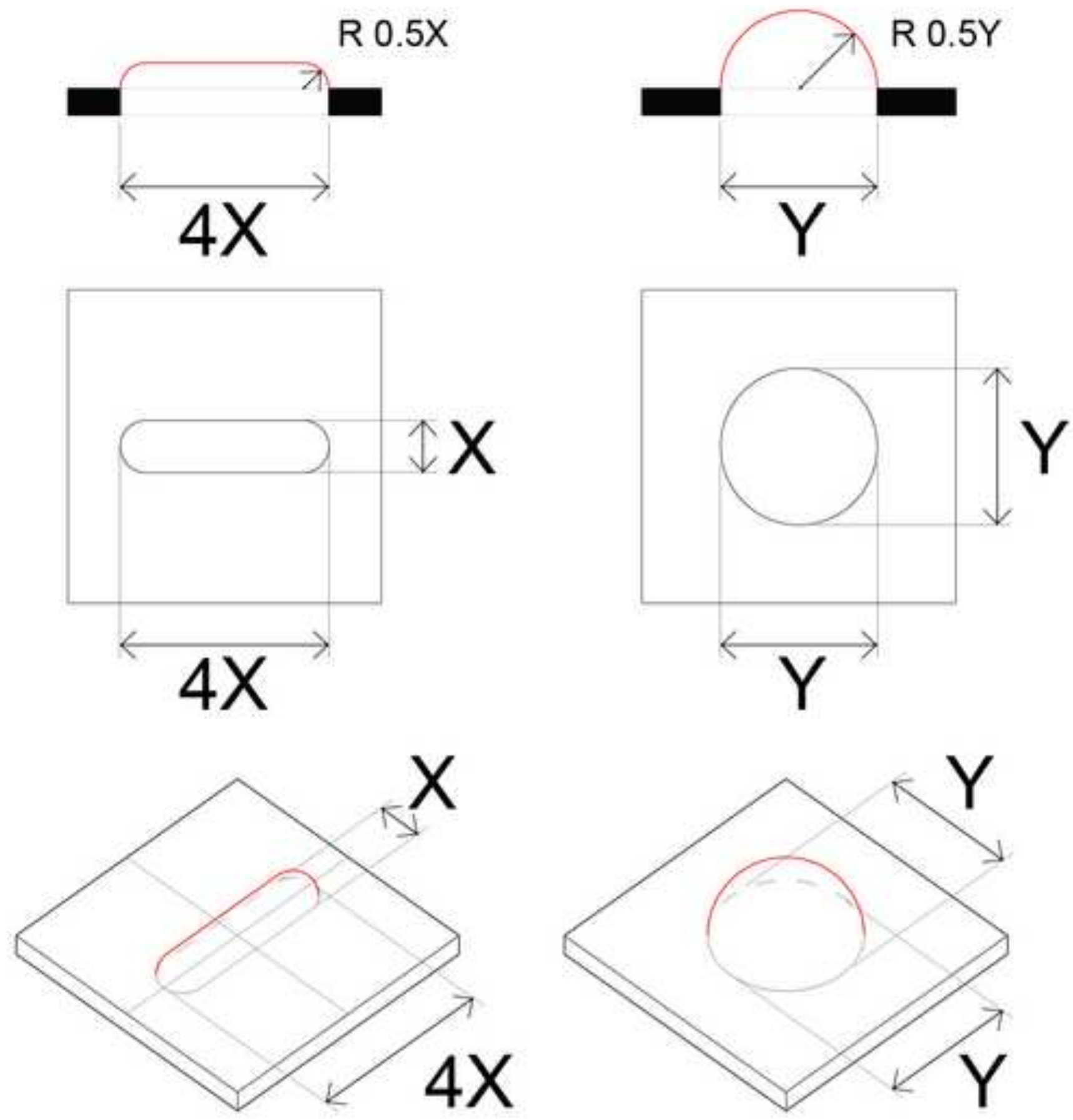
Click here to download high resolution image
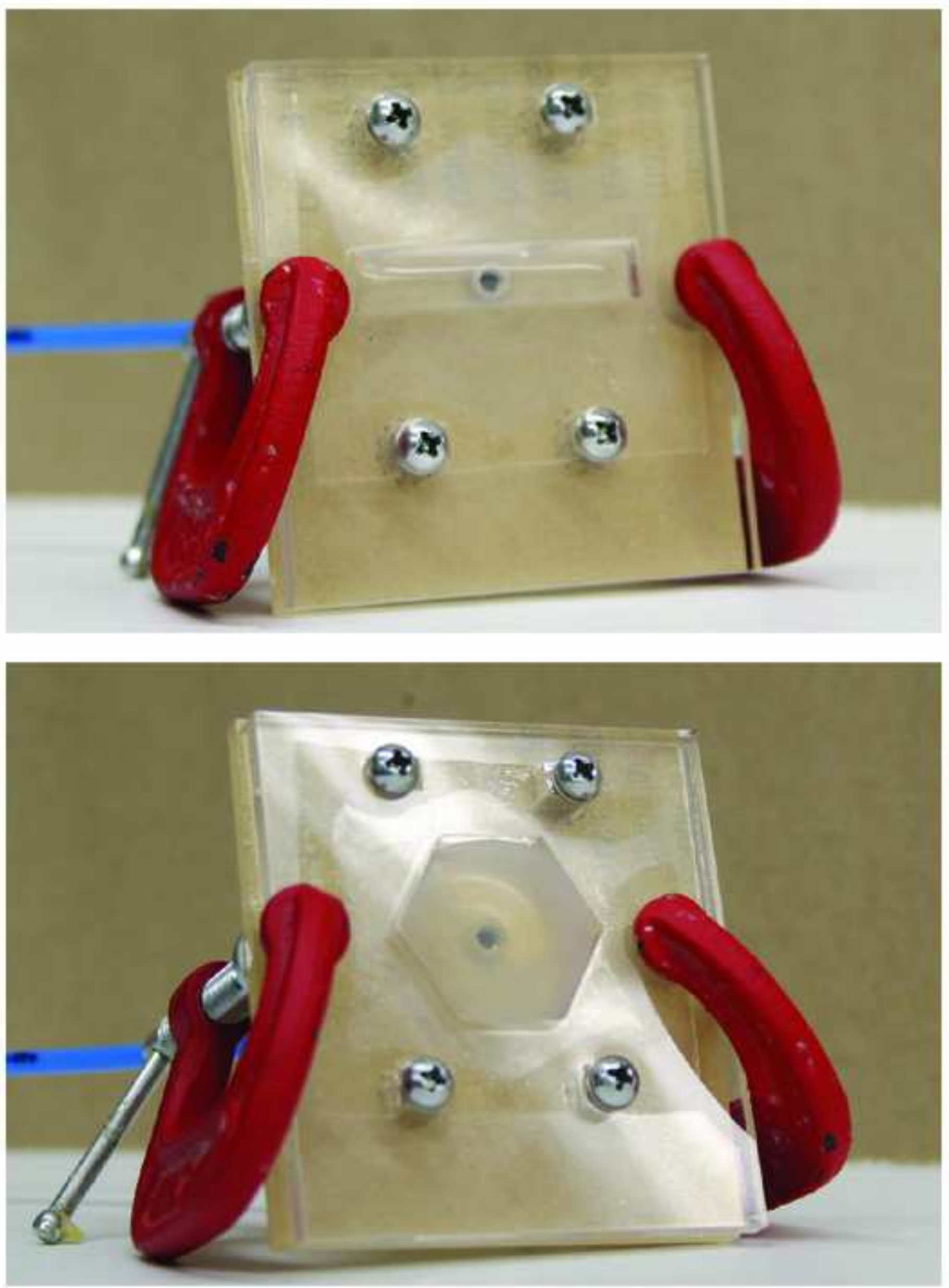
Click here to download high resolution image

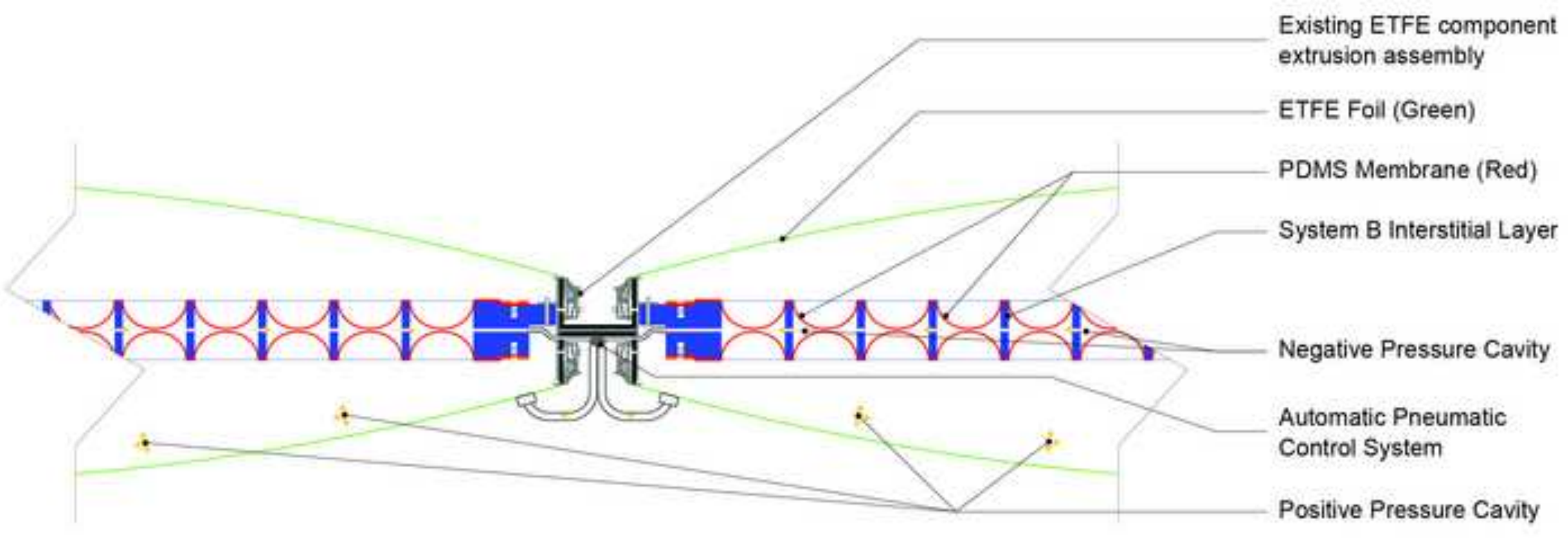

Existing ETFE component 